\title{
Die Dommusik zu St. Stephan im 18. Jahrhundert - Institutionsgeschichte
}

\section{Musical Life at the St. Stephen's Cathedral in Vienna during the 18th Century - Institutional History}

Helena Kramářová / helena.kramarova@gmail.com

Department of Musicology, Faculty of Arts, Masaryk University, Brno, CZ

\begin{abstract}
The Cathedral of St. Stephen in the 18th century was one of the most important music institutions in Vienna. Thanks to source research, it was possible to discover new insights into the music ensembles in St. Stephen's Cathedral. The contribution also brings new information about the music during the liturgical year and special occasions. It focuses on the purchase of musical instruments and compositions for the cathedral, too.
\end{abstract}

\section{Keywords}

Vienna, Cathedral of St. Stephen, 18th century, Georg Reutter, Leopold Hofmann, Johann Georg Albrechtsberger, the liturgical year, organs, musical instruments, purchase of compositions 
Die Kirche St. Stephan zu Wien erfreute sich im Laufe ihrer Geschichte großer Bedeutung und nimmt bis heute eine Sonderstellung als Stadtpfarr- und gleichzeitig Domkirche unter Wiens geistlichen Einrichtungen ein, was sich auch im regen Musikbetrieb widerspiegelt. ${ }^{1}$ Die Dommusik stand im 18. Jahrhundert der Hofmusik sehr nahe und unter ihren Vertretern befanden sind nicht nur bedeutende Komponisten, sondern insbesondere auch bekannte Sänger und Musiker der damaligen Zeit. Um sich mit der reichhaltigen Wiener Musikkultur und vor allem der Kirchenmusik im 18. Jahrhundert zu beschäftigen, ist es unabdingbar, den Musikbetrieb der Hauptkirche der Wiener Erzdiözese genauer in Augenschein zu nehmen.

Neue Erkenntnisse zum Musikbetrieb im Stephansdom lassen sich durch eine Neuauswertung der Quellenbasis gewinnen. Eine gründliche Auseinandersetzung und eine kontrastive Betrachtung der heute nur zum Teil überlieferten Dokumente des Kirchenmeisteramts ermöglichen es, verschiedene Fragestellungen zu betrachten, die den damaligen Musikalltag im Dom genauer darlegen können. Trotzdem schöpfte die bisherige Fachliteratur zum Musikgeschehen im Stephansdom hauptsächlich aus den in der ersten Hälfe des 19. Jahrhunderts entstandenen Fachtexten. ${ }^{2}$

Im folgenden Artikel werden nach der Darstellung der Domkapellen weitere Punkte berücksichtigt, die überwiegend mit dem alltäglichen Geschehen im Dom zusammenhängen. Nicht beachtet werden dabei die Tätigkeit der Hofkapelle im Dom und Gottesdienste der Cäcilien-Bruderschaft, weil diese an der Michaeler Kirche ansässig war. ${ }^{3}$

$\mathrm{Zu}$ den wichtigsten Bereichen, die neu bewertet werden müssen, gehört der alltägliche und außergewöhnliche Musikbetrieb in der Domkirche zu St. Stephan während des 18. Jahrhunderts und die Frage, an welchen Orten damals tatsächlich musiziert wurde. Von Bedeutung ist ebenfalls, welche Musikinstrumente den Dommusikern zur Verfügung standen und was sich anhand der Archivalien über Anschaffung der Musikalien

1 Die Entstehung dieses Textes wurde durch das Stipendium AKTION (CZ-AT) ermöglicht. Ein aufrichtiger Dank für zahlreiche Ratschläge gilt Prof. Dr. Otto Biba.

2 Zu nennen sind: ZSCHOKKE, Hermann. Geschichte des Metropolitan-Capitels zum Heiligen Stephan in Wien (nach Archivalien). Wien, 1895; UHLIRZ, Carl. Die Rechnungen des Kirchenmeisteramts von St. Stephan zu Wien. Wien: Braumüller, 1901-1902; BRUNNER, Hans. Die Kantorei bei St. Stephan in Wien. Wien 1948; HASELBÖCK, Hans. Sechs Jahrhunderte Orgelbau im Wiener Stephansdom. In HASELBÖCK, Hans. Von der Orgel und der Musica Sacra. Historisch-kritische Beiträge zu Fragen von Orgelbau, Orgelkomposition und neuer Kirchenmusik. Wien-München, 1988; SEIFERT, Herbert. Die Entfaltung des Barocks, In FLOTZINGER, Rudolf - GRUBER, Gernot. (Hr.) Musikgeschichte Österreichs. Bd. 1, S. 299-361.

3 In den Ausgaben aus der Kirchenkassa zu St. Stephan sind bemerkenswerter Weise in keinem Jahr Ausgaben für das Cäcilien-Fest der Cäcilien-Buderschaft zu finden. Dass das Fest aus anderen Mitteln bezahlt werden musste, bezeugt die Ausgabe, resp. eine Rückzahlung aus dem Jahr 1748. Dem „löbl: Musicanten CongregationsSecretaio Leopold Christian wegen ihren gehaltenen Caeciliafest [...] annoch brauchbahren Wax 15 fl. 36x. bezahlt" wurde. Auf Basis dieser Aussage lässt sich vermuten, dass nicht nur Wachs, sondern auch andere Ausgaben wie Musikkosten von der Cäcilien-Bruderschaft finanziert wurden, die ihren Sitz nicht bei der Domkirche hatte. Das war höchstwahrscheinlich der Grund dafür, warum die „Congregation deren herrn Musicanten wegen ihren gehaltenen Caecilia Fest den Gebühr 5 fl." in die Domkasse zahlen musste. Vgl. Wiener Stadt- und Landesarchiv (weiter nur: AT-WStLA), Handschriften (weiter: HS), A/41.25 Inventar von St. Stephan 1751; Für ausführlichere Informationen zu Musikbruderschaften siehe: HILSCHER, Elisabeth. Musikbruderschaften als Karrierenetzwerke für Stadt und Hof in Wien. In LOBENWEIN, Elisabeth - SCHEUTZ, Martin - WEISS, Alfred Stefan (Hg.). Bruderschaften als multifunktionale Dienstleister der Frühen Neuzeit in Zentraleuropa. Wien: Böhlau, 2018, S. 357-372. 
sagen lässt, die heute zum großen Teil nicht mehr vorhanden sind. Durch die lange Zeitspanne eines Jahrhunderts können Zusammenhänge und Traditionen sowie ansonsten kaum merkbare Veränderungen und Umstrukturierungen besser verfolgt und im breiteren Kontext dargestellt werden.

\section{Die Dommusikkapellen}

Der liturgische und somit auch musikalische Betrieb in der Domkirche zu St. Stephan war sehr rege und aufwändig, daher gestalteten den musikalischen Rahmen zu verschiedenen Anlässen mehrere Ensembles. Die meisten Angaben über Ensembles und einzelne Musiker sind in den Rechnungsbüchern und im überlieferten Aktenmaterial des Kirchenmeisteramtes zu finden. Die Rekonstruierung der einzelnen Kapellen erschwert jedoch die Tatsache, dass Sänger und die Instrumentalgruppe der Posaunisten von 1702 bis in die 1770er Jahre meistens summarisch angegeben wurden. Hingegen sind andere Instrumentalisten sowie Bezieher von Sonderzahlungen für extra geleistete Dienste namentlich angeführt. Erst seit 1781 wurden Rechnungen für jeden Sänger und Musiker einzeln ausgestellt. Die Abrechnungen sind ab diesem Zeitpunkt so detailliert, dass nachvollzogen werden kann, ob es sich bei dem jeweiligen Musiker beispielsweise um den ersten oder zweiten Violinisten handelt. ${ }^{4}$ Dank dieser Quellengrundlage lässt sich bestimmen, dass im Dom die Essentialkapelle und die Gnadenbildkapelle tätig waren. Überdies wird ersichtlich, inwiefern sich die Personalbasis beider Kapellen überschnitt und wie die Musiker beider Kapellen untereinander kooperierten. Separat werden die Trompeter und Pauker behandelt, die im Dom mitwirkten.

\section{Essential- und Gnadenbildkapelle}

Die „Haupt““ oder „Essentialkapelle“ leitete im Stephansdom ein Domkapellmeister. Im 18. Jahrhundert bekleideten diese Stelle Michael Zächer, Johann Joseph Fux, Georg Reutter d. Ä., und Georg von Reutter d. J. Nach ihm übernahm Leopold Hofmann und seit 1794 Johann Georg Albrechtsberger die Leitung der Kapelle. ${ }^{5}$ Dem jeweiligen Domkapellmeister stand ein Organist zur Verfügung. Die Essentialkapelle bestand während des 18. Jahrhunderts aus sechs Sängerknaben, höchstens neun Choralisten und Instrumentalisten. Hinzu kam noch ein Subcantor, der für Leitung der Choralmusik verantwortlich war. Dies war in der Regel ein angestellter Choralist oder Instrumentalist.

4 Die einzelnen biographischen Angaben anhand dieser Quellen werden im Rahmen der derzeit entstehenden Dissertation der Autorin veröffentlich. Das Dissertationsprojekt heißt vorläufig: „Der Kirchenmusikalltag im späten 18. Jahrhundert am Beispiel von Brünn und Wien," Masaryk-Universität, Brünn, [Einreichtermin: Januar 2020].

5 Vgl. BRUNNER, Hans. Die Kantorei bei St. Stephan in Wien: Beiträge zur Geschichte der Wiener Dommusik. Wien: Verl. Albrecht Dürer, 1948. 
Beispielsweise ging es um Mathias Ignaz Feichtwöck; er ist zwischen den Jahren 1709 und 1721 als Tenorist bei St. Stephan belegt. Im Jahr 1728 wurde er als Subcantor erwähnt. ${ }^{6}$ Es ist anzunehmen, dass er gleichzeitig als Tenorist und Subcantor tätig war.

Grundsätzlich waren die Choralisten zusammen mit den Sängerknaben dazu verpflichtet, sich sowohl an der Choral- als auch an der Figuralmusik zu beteiligen. Die Besetzung der Vokalstimmen war folgende: vier der Kapellknaben sangen Cantus, zwei Knaben mit einem erwachsenen Sänger sangen Alt; Tenor und Bass wurden jeweils durch drei Erwachsene besetzt. Im Jahre 1773 handelte es sich um sechs Sängerknaben ${ }^{7}$, den Altisten Anton Pacher, die drei Tenoristen Ferdinand Hofmann, Leopold Panschab und Joseph Krottendorfer sowie die drei Bassisten Ferdinand Schallhaß, Tobias Gsur und Joseph Stadtler. ${ }^{8}$ In Abhängigkeit des Kirchenjahres konnte die Besetzung variieren: In der Adventszeit wurde der Sängerkreis um einen Tenoristen und Bassisten erweitert. Sie bekamen zusammen mit dem angestellten erwachsenen Altisten eine Sonderzahlung für die Rorate. Diese Struktur blieb über das ganze 18. Jahrhundert hinweg unverändert.

Im Gegensatz dazu veränderte sich die Anzahl der Instrumentalisten deutlich, die zur Essentialkapelle gehörten. Zwischen den Jahren 1702 und 1727 standen fünf Streichinstrumentenspieler (zwei Violinisten, zwei Gambisten, ein Violonist) und ein Posaunist zur Verfügung. In der Folgezeit kam es zu einer allmählichen Vergrößerung des Instrumentalensembles: Im Jahr 1728 stieg die Anzahl der Streicher auf sieben, 1731 kam ein Kornettist ${ }^{9}$ (Zinkenbläser) hinzu. Bis in das Jahr 1736 waren sieben Violinisten am Dom tätig. Ein weiterer Ausbau der Kapelle fand 1739 statt. Aus den Erträgen der Vestenburglichen Stiftung ist ableitbar, dass zwei Violinisten à $40 \mathrm{fl}$ und ein Bassist mit $50 \mathrm{fl}$ jährlich bezahlt wurden..$^{10} 1743$ waren elf Violinistenstellen in der Essentialkapelle besetzt. Ab 1750 bewegte sich die Anzahl der Violinisten zwischen zehn bis zwölf Mitgliedern. Vom Stiftungsüberschuss wurde ab dem Jahr 1765 ein weiterer Violinist ${ }^{11}$ aufgrund des Dekrets vom 11. Juni 1765 für ,die all wochentlich gehaltenen Corporis Christi Ämbtern pr: jährlich $24 \mathrm{fl}^{\text {" }}$ bezahlt. ${ }^{12}$

Die zweite regelmäßig im Stephansdom aktive Kapelle war die „Gnadenbildkapelle“, deren Dienste mit dem liturgischen Betrieb beim Gnadenbild Maria Pötsch verbunden waren. Als im Dezember 1697 das Gnadenbild in den Dom übertragen wurde, versah höchstwahr-

\footnotetext{
6 Vgl. Domarchiv St. Stephan (weiter AT-DAWST), Kirchenmeisteramt von St. Stephan (weiter: KMA), Rechnungsbuch 1728, Fol. 49v.

7 Ihre Identität wurde mit wenigen Ausnahmen in den Rechnungen nie angegeben.

8 AT-DAWST, KMA, Rechnungsbuch 1773, Fol. 101ff.

9 DICKEY, Bruce. Far buon stromento: A Survey of the Technique and Repertoire of the Cornetto in its Golden Age. In HAAG, Sabine. (Hrsg.) Die Zinken und der Serpent der Sammlung Alter Musikinstrumente. Bergkirchen, Edition Bochinsky, 2011, S. 68-89.

10 Im Jahre 1766 wurden auch diese Beträge herabgesetzt; die Violinisten bekamen à $32 \mathrm{fl}$ und der Bassist $40 \mathrm{fl}$ jährlich - der ganzen Musik wurde nur $80 \mathrm{fl}$ bezahlt.

11 Diese Stelle entspricht später der 12. Violinisten-Stelle.

12 AT-DAWST, KMA, Rechnungsbuch 1765, Fol. 132v-133r.
} 
scheinlich die gesamte Essentialkapelle zusammen mit den Trompetern die musikalischen Dienste, wie es sich anhand von Rechnungen aus dem Jahre 1702 ableiten lässt. ${ }^{13}$

Für die weitere Entwicklung der Gnadenbildkapelle waren folgende Ereignisse von Bedeutung: Erstens war im Jahre 1707 die sog. Kurzische Stiftung angelegt worden, deren Erträge für die Bezahlung der großbesetzten Musik mit Trompeten und Pauken bei den gehaltenen Gottesdiensten an allen Sonn- und Feiertagen dienen sollte. ${ }^{14}$ Dank der Stiftung war es möglich, die Hoftrompeter gegen einen jährlichen Betrag von $600 \mathrm{fl}$ einzusetzen. Zweitens bekam die Gnadenbildkapelle ihren eigenen Kapellmeister; spätestens ab 1709 wurde ihm für die Versorgung von drei Kapellknaben ein sog. Kostgeld von $600 \mathrm{fl}$, sowie die Zimmerbehilf von $60 \mathrm{fl}$ jährlich ausgezahlt. ${ }^{15}$ Drittens wurde dem Gnadenbild-Kapellmeister ein Adjunktum, eine offizielle, jährliche Entlohnung bezahlt. Dies ist erstmals für das Jahr 1727 belegt: „dem Herrn Johann Georg Reinhardt Capellmeister bey den Bözer gnaden Bildt Unser Lieben Frauen sein Erstes quartal adjucta gebühr vom $1^{\text {ten }}[$ Octo]bris bis ult [im]a [Dezem]bris 727 laut dessen Bescheinigung N. 240 zahlt zum ersten mahlen 12 fl $30 x .{ }^{\prime \prime} 16$

Zur ersten Umstrukturierung der Gnadenbildkapelle kam es in den 1750er Jahren. In der Zeitspanne von 1754 bis 1757 durften wegen des kaiserlichen Verbots keine Trompeter im Dom mitwirken, darum wurden aus den Mitteln der Kurzischen Stiftung für sog. Kurzische Musizi - vier Geiger - angestellt. ${ }^{17}$ Im letzten Quartal des Jahres 1757 wurden wieder Hoftrompeter für die Mitwirkung bezahlt; ihre Besoldung sank dabei auf 119 bis $184 \mathrm{fl}$ von bisher $600 \mathrm{fl}$. Die Anzahl der kurzischen Musizi stabilisierte sich in den 1760er Jahren auf vier Violinisten und einen Violonisten. Bis ins Jahr 1771 erhielt der Gnadenbildkapellmeister die Gehälter der Sänger und Musiker der Gnadenbildkapelle summarisch, mit Ausnahme der Kurzischen Musizi und der Hoftrompeter. Daher kann nicht exakt bestimmt werden, wie viele Sänger und Musiker aus der Essentialkapelle mitspielten. Eine präzisere Führung der Buchhaltung ab den 1780er Jahren gibt darüber jedoch später Auskunft. Folgendes Schema veranschaulicht die oben beschriebenen Veränderungen und den Zusammenhang beider Ensembles:

13 AT-WStLA, HS, A41/20 Kirchenmeisteramts-Rechnungen von St. Stephan -1702, Fol. 108r.

14 AT-WStLA, Hauptarchiv, Akten und Verträge: A1/9/1707, Testament des hofbefreiten Handelsmannes Michael Kurz.

15 Beim Eintritt Georg Reutters d. Ä. an die Domkapellmeistersstelle übernahm er beide Zahlungen für das Knabenkostgeld der Kapellmeister der Essential-Kapelle, da die Kapellknaben unter seiner Aufsicht waren. Vgl. HOCHRADNER, Thomas - VÖSÖMARTY, Géza Michael. Zur Musikpflege am Altar Mária Pócs (Maria Pötsch) in St. Stephan in Wien. In Studia Musicologica Academiae Scientiarum Hungaricae, 2000, T. 41, Fasc. 1/3, S. 156-164.

16 AT-DAWST, KMA, Rechnungsbuch 1727, Fol. 48r.

17 Vgl. HOCHRADNER - VÖSÖSMARTY, op. cit., S. 158. 


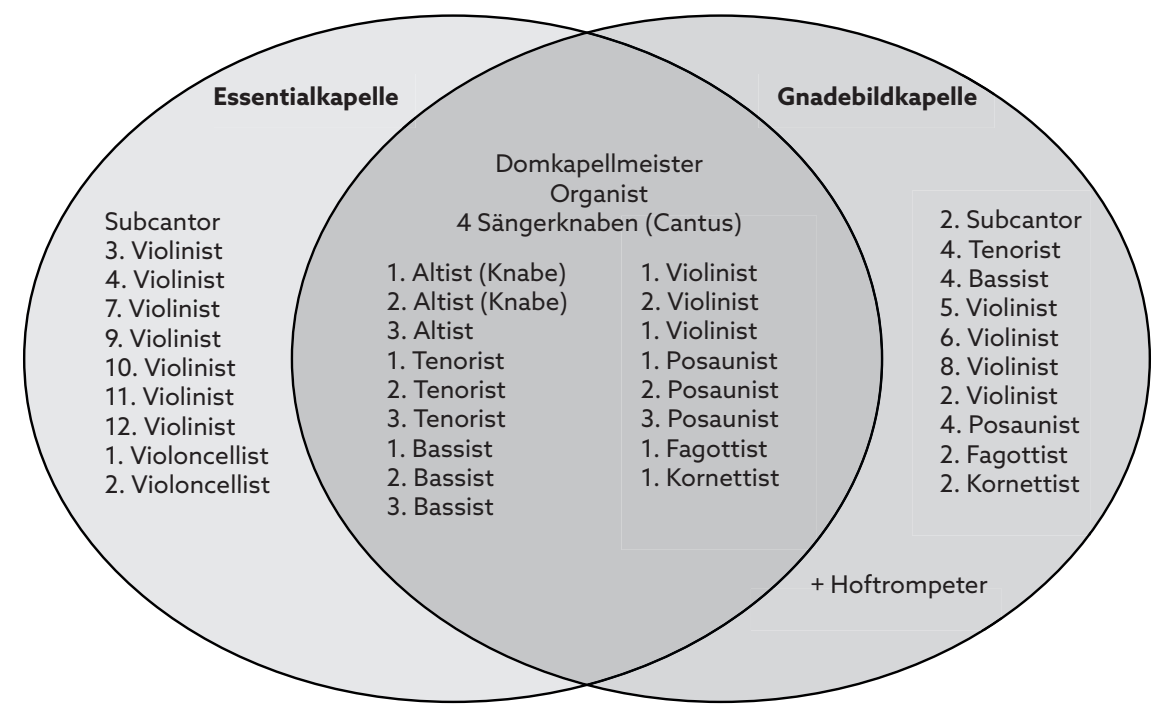

Grafik 1 Besetzung der Domkapellen im Jahr 1782

Die Grafik zeigt, wie eng beide Kapellen hinsichtlich ihrer Personalbasis zusammenhingen. So wurde auch die oben angesprochene Stelle des Gnadenbildkapellmeisters nach dem Tod von Ferdinand Schmidt von 1757 bis 1772 nicht besetzt. Das Geld für das ganze Ensemble übernahm in dieser Zeit der Domkapellmeister Georg von Reutter, jedoch ohne als Gnadenbildkapellmeister bezahlt zu werden. Seit 1773 wurde sein Nachfolger Leopold Hofmann sowohl als Domkapellmeister, als auch Gnadenbildkapellmeister bezahlt. Die Unterschiede beider Kapellen lagen, neben der Größe der Besetzung, vor allem in den Finanzierungsquellen der Kapellen; die Essentialkapelle wurde aus der Kirchenkasse und die Gnadenkapelle aus den eigenen vom Magistrat zur Verfügung gestellten Mitteln bezahlt.

\section{Trompeter und Pauker}

Als eine besondere Instrumentalgruppe am Stephansdom müssen die Trompeter und Pauker behandelt werden, denn sie wurden am Anfang des Jahrhunderts zeitgenössisch nicht als sogenannte Essentialisten betrachtet. Die Trompeter und Pauker hatten unter Musikern eine besondere Stellung, da sie hauptsächlich für zeremonielle und repräsentative Dienste am Hof oder in der Stadt (z. B. bei der Hoftafel des Kaisers) tätig waren. ${ }^{18}$ Deshalb war es die Regel, dass diese nicht zu den Mitgliedern der Kirchenmusikensembles zählten. Dies ist auch der Grund, warum sie in den Quellen getrennt von den anderen Musikern aufgeführt werden.

18 Vgl. ŠTĚDROŇ, Bohumír. Společenské úkoly hudby v 18. století. [Gesellschaftliche Funktionen der Musik im 18. Jahrhundert.] In Časopis Matice moravské 69, 1950, S. 312. 
Häufig finden sich in den Archivalien Zahlungen an die Hoftrompeter, da sie bei speziellen Anlässen separat bezahlt wurden. Es handelt sich dabei um Zahlungen für Dienste in der Essentialkapelle bei den hohen Festen sowie für das Spielen, „wann Ihre hochfürstliche Eminenz selbsten pontifizieren"19. Diese Zahlungen sind in den Quellen unter Extra-Ausgaben angegeben. Was sie bei dieser Gelegenheit spielten, ist leider nicht verzeichnet. Die Präsenz der Hoftrompeter an sich weist also bereits auf eine außerordentliche kirchliche Feierlichkeit hin. Das Hoftrompeter-Ensemble musizierte auch zusammen mit der Gnadenbildkapelle.

Auch in der städtischen Musikkultur waren Trompeter, meist als Turner oder Türmer bezeichnet, verankert. Sie unterstanden dem Türmermeister, der beispielsweise in Wien zum einen das sogenannte Turm anblasen - das Musizieren auf dem St. Stephansturm und im Dom - und zum anderen die Turmwache verantwortete. ${ }^{20}$ Für Musikdienste standen dem Turmmeister Musiker und Gesellen zur Verfügung; die Anzahl der Instrumentalisten belief sich im 18. Jahrhundert auf fünf Personen. Dazu kam noch ein sechster Musiker, ein Posaunist, resp. Fagottist. Die Ausgaben für die Türmer wurden bis 1760 unter der Rubrik „Ausgaben auf St. Stephans Turm und Uhr“ erfasst.

Obwohl die Trompeter nicht offiziell Teil der Essentialkapelle sein durften, wurde diese Regelung im Laufe des Jahrhunderts nicht mehr so streng eingehalten. Ab 1761 sind die Entlohnungen der Instrumentalisten nicht mehr in einer eigenen Rubrik, sondern unter den Ausgaben „Auf die Cantorey der ordinari Vocal- und Instrumental Music“ zu finden. Die Besetzung des Ensembles im 18. Jahrhundert lässt sich anhand der Rechnungen aus den 1780er Jahren bestimmen: es handelte sich um zwei Kornettisten, drei Posaunisten, einen Fagottisten. Diese Musiker wurden zu Diensten bei der Essentialkapelle herangezogen. Zwei weitere, nämlich der vierte Posaunist und der zweite Fagottist bildeten im Regelfall die Verstärkung für die Gnadenbildkapelle. ${ }^{21}$

Überdies war es üblich, dass Musiker verschiedene Musikinstrumente beherrschten, was natürlich den exakten Nachvollzug erschwert, wer, wann welchen Dienst verübte. Wurde dennoch ein zusätzliches Musikinstrument, beispielsweise im Bereich der Holzbläser benötigt, und befand sich im Personalstand kein entsprechender Musiker, so war es problemlos möglich, einen Substituten ${ }^{22}$ zu engagieren.

Ein weiteres, mit der Dommusik verbundenes Trompetenensemble, waren die landesfürstlichen Musiker, die so genannten „Landschaftstrompeter". ${ }^{23}$ Belege über deren

19 AT-DAWST, KMA, Rechnungsbuch 1748, Fol. 87r.

20 Die Funktionen der städtischen Musiker unterlagen während des 18. Jahrhunderts bedeutende Veränderungen, deren Erforschung noch ein Desiderat darstellt. Am Ende des Jahrhunderts wurde beispielsweise in Brünn (CZ) heftig diskutiert, ob ein neuer Stadtturmmeister benannt werden soll oder ob diese Funktion auch angesichts der Kirchendienste längst überflüssig sei. Dieser Frage wird im Rahmen des Dissertationsprojekts der Autorin nachgegangen. $\mathrm{Zu}$ den Pflichten von Stadtmusikern am Beispiel von Brünn siehe die Instruktion aus dem Jahre 1803: D’ELVERT, Christian. Geschichte der Musik in Mähren und Oesterr.-Schlesien. Brno, 1873, S. 163.

21 Siehe Besoldungen vor und nach der Musikreform im Vergleich. In KRAMÁŘOVÁ, Helena. Die Dommusik zu St. Stephan im 18. Jahrhundert in sozialgeschichtlicher Perspektive. In Musicologica Brunensia, 54, 2019, Nr. 1, S. 173-195.

22 Siehe Orte des Musizierens.

23 Vgl. ŠTĚDROŇ, Bohumír. Zur Entwicklungsgeschichte einer unbekannten Musik-Gesellschaft im 17. und 18. Jhdt. In Die Musikforschung 21, 1968, s. 438-458. 
Mitwirkung bei den Feierlichkeiten im Dom zu St. Stephan lassen sich erst um das Jahr 1800 nachweisen. ${ }^{24}$ Dennoch ist es möglich, dass sie bereits früher bei z. B. Prozessionen mitgewirkt haben und aus einer anderen Quelle bezahlt wurden. Da sie nicht regelmäßig Dienste am Dom zu St. Stephan versahen, wird nicht näher auf sie eingegangen.

\section{Orte des Musizierens}

Im Stephansdom sind gleich mehrere Orte für das Musizieren bekannt, die anhand der überlieferten Quellenbasis wenigsten in Teilen genannt werden können. Es handelt sich dabei um die Totenkapelle, der Musikchor, der Chor oberhalb der unteren Sakristei und die Westempore.

Die Totenkapelle der Domkirche verfügte über ein Positiv, das im Jahre 1746 vom bürgerlichen Orgelmacher Johann Hencke ${ }^{25}$ verfertigt wurde. Sie wurde im gleichen Jahr noch mit einem neuen Altarblatt versehen, das vom kaiserlichen Theatermaler Anton Danne geliefert wurde. ${ }^{26}$ Das Positiv durfte für die Musik bei den Einsegnungszeremonien benutzt werden. ${ }^{27}$

In den Dominventaren wird häufig der hölzerne, im Jahre 1701 neu erbaute „Musikanten-Chor“28, bzw. „Musik-Chor“ ${ }^{29}$ erwähnt, auf dem sich die Musikinstrumente der Metropolitankirche befanden. Dieser Chor wurde gegenüber des Kaiseroratoriums erbaut und erst durch den Dombrand am 12. April 1945 zerstört. Auf dem Chor befand sich auch eine kleine Orgel, die der Disposition nach zur Begleitung der Figuralmusik bestimmt war. ${ }^{30}$ Eine weitere Orgel stand auf dem Chor oberhalb der unteren Sakristei auf dem Füchsel-Baldachin. Dieser Chor befand sich gegenüber des Pilgram-Orgelfußes.

Auf der Westempore stand die große Orgel (u. a. Festorgel genannt), die allerdings in erster Linie nicht zur Aufführung der Figuralmusik bestimmt war, sondern zum Einund Auszug, sofern nicht ein Trompeterchor blies, aber auch wenn er abwechselnd mit

24 Siehe Kapitel 2.1 Allgemeine liturgische Feste.

25 HOPFNER, Rudolf. Hencke (Henke, Henckl, Hengel), Johann. In Oesterreichisches Musiklexikon online [online], 20XX [2019-03-13] URL: 〈http://www.musiklexikon.ac.at/ml/musik_H/Hencke_Johann.xml>.

26 AT-DAWST, KMA, Rechnungsbuch 1746, Fol. 57v; Im Falle des Bildhauers handelte sich wahrscheinlich um Johann Georg Hildebrand (1667-1747), der das Altar für die Elisabethinenkirche schuf. Vgl. CZEIKE, Felix. Historisches Lexikon Wien. Wien: Verlag Kremayr \& Scheriau, 1994.

27 AT-DAWST, KMA, Rechnungsbuch 1746, Fol. 52r.

28 AT-WStLA, HS, A/42/2 Inventar von St. Stephan 1732, Fol. 118r.

29 AT-DAWST, Inventar 1788, Fol. 58r.

30 Für nähere Angaben zur Disposition aller erwähnten Orgel siehe: ALLMER, Gottfried. Orgeln und Orgelbau in der Dom- und Metropolitankirche St. Stephan in Wien. In Das Orgelforum 14, 2011, S. 32-67. 


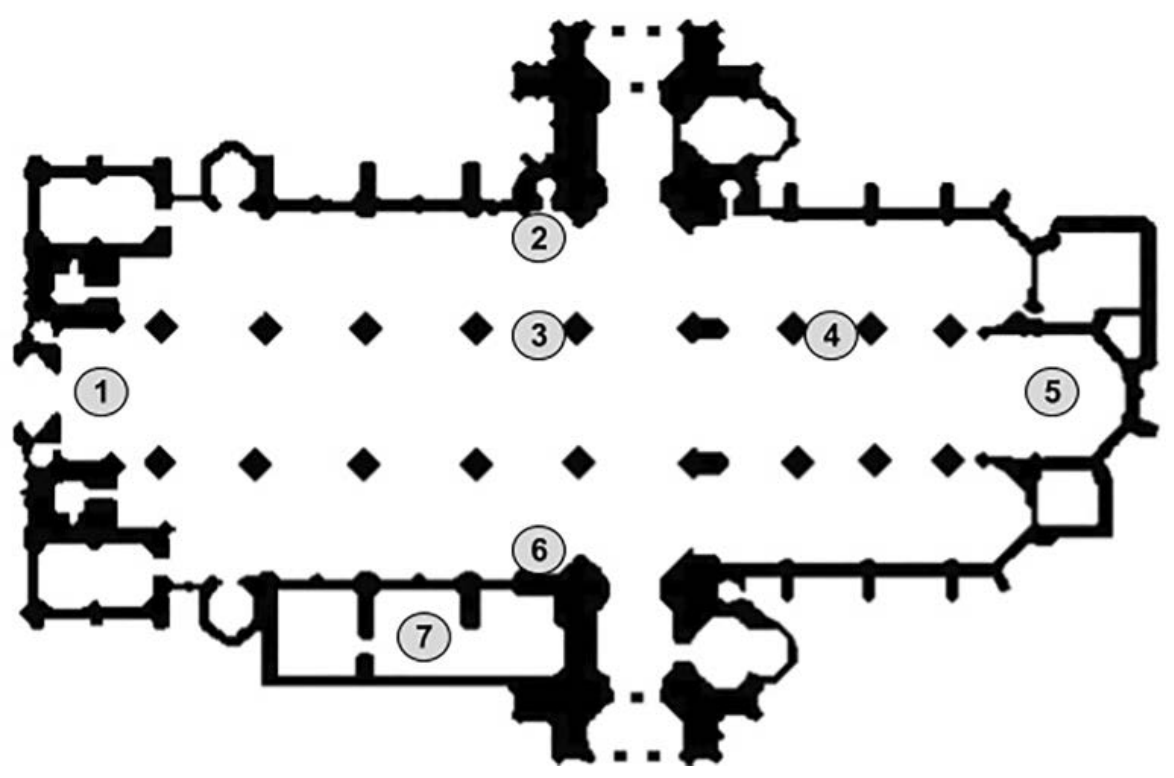

Bd. 1 Vereinfachter Grundriss - Stephansdom: Nr. 1 - Westempore, Nr. 2 - Pilgram' Orgelfuß, Nr. 3 - Altes Gnadenbild (Maria in der Sonne), Nr. 4 - Musikchor, Nr. 5 - Hochaltar,

Nr. 6 - Füchsel-Baldachin, Nr. 7 - Untere Sakristei.

der Orgel spielte. ${ }^{31}$ Das nicht regelmäßige Spielen bei der großen Orgel geht aus einem Brief des Domkapellmeisters Albrechtsberger vom 17. Oktober 1793 hervor. $^{32}$

Allem Anschein nach wurde an den Domkapellmeister Johann Georg Albrechtsberger der Wunsch des Magistrats herangetragen, wenigstens viermal im Jahr die gesamte Kirchenmusik von der Westempore aus erklang. Das betrifft die Hochämter am St. Leopoldi-Tag, zu Weihnachten, Ostern und Pfingsten. Seines Erachtens ist aber die „ordinari St. Stephans Musik hierzu zu schwach." Das Ensemble hatte im Jahre 1793 laut vorliegender Rechnungen 36 bezahlte Sänger und Musiker (inkl. Sängerknaben und bezahlten Adjunkten). Nichtdestotrotz war es nach Albrechtsbergers Meinung - wahrscheinlich aufgrund ungünstiger Akustik - notwendig, weitere 24 Personen für die genannten Festtage anzustellen. Als Anhang seiner Bitte wurde von Johann Georg Albrechtsberger folgende Liste hinzugelegt:

31 Vgl. EBENBAUER, Melitta. Zur Geschichte der Dommusik. [online] URL: < http://www.dommusik-wien. at/Dommusik/media/ZurGeschichtederDommusik_Ebenbauer.pdf>; HASELBÖCK, Hans. Sechs Jahrhunderte Orgelbau im Wiener Stephansdom. In HASELBÖCK, Hans. Von der Orgel und der Musica Sacra. Historisch-kritische Beiträge zu Fragen von Orgelbau, Orgelkomposition und neuer Kirchenmusik. Wien-München, 1988

32 AT-DAWST, KMA, Erledigungen 1792-1800, Kart. 8/1, Fol. 726. 


Personale $^{33}$
Einer verstärkten Musik zum Leopoldi- oder anderen Fest bei der großen Orgel:
2 Sopranisten
2 Altisten Notandum: wenn keine Sänger zum Respondiren auf den kleinen Chor gestellt werden
2 Tenoristen
2 Bassisten
7 Violonisten samt 3 Violen
1 Bassetlist
1 Violonist
2 Hoboisten
2 Flautraversisten
2 Trompeter annoch, und
1 Pauker, weil am Leopoldi fest nur 2 Clarinibläser vorgeschrieben sind, NB Si placet
Summa 24 der Personen

Im Anschluss folgt die Kostenberechnung, also welchen Betrag die einzelnen Sänger und Musiker für ihre Dienste erhalten sollten. Die Gesamtkosten pro Festtag würden somit 13 fl 46 x betragen; eine Summe, die laut Albrechtsberger ganz gering sei.

Der Magistrat bewilligte Albrechtsberger für die genannten vier Hochämter die Verstärkung der Musik, damit auf dem großen Chor musiziert werden konnte. Bereits im Jahre 1793 betrug die Ausgabe „für zwei mit verstärkter Musik gehaltene Ämter"34 $29 \mathrm{fl} 32$ x, was einer etwas höheren Summe für ein Amt entspricht, als Albrechtsberger ursprünglich beantragt hatte. Eine letzte Angabe diesbezüglich stammt aus dem Jahre 1794, aus der hervorgeht, dass für die Musikverstärkung an den Festtagen $55 \mathrm{fl} 4$ x ausgegeben wurde..$^{35}$

All das beweist die gängige Praxis, dass die meisten Messen vom Musikchor aus musikalisch begleitet wurden, die Kapellen jedoch auch auf spätestens seit 1793 der Westempore neben der großen Orgel spielten, obschon mit Verstärkung und nur vier Mal pro Jahr.

\section{Musik im Dom im Laufe des liturgischen Jahres}

Um die Ausgaben auf die Kirchenmusik während des 18. Jahrhunderts bewerten zu können, ist es notwendig zu veranschaulichen, bei welchen Gelegenheiten die Musiker der Essentialkapelle und der Gnadenbildkapelle sich musikalisch beteiligten. Der rege Musikbetrieb macht dann ersichtlich, warum es für die Domkirche nötig war, zwei Musikensembles zu unterhalten. Der regelmäßig verrichtete Musikbetrieb wurde im Laufe des 18. Jahrhunderts (bis 1784) nur selten verändert. Die Musikdienste während des liturgischen Jahres - auf welche später noch näher eingegangen wird - lassen sich in zwei Kategorien

33 AT-DAWST, KMA, Erledigungen 1792-1800, Kart. 8/1, Fol. 726.

34 AT-DAWST, KMA, Rechnungsbuch 1793, S. 61.

35 AT-DAWST, KMA, Rechnungsbuch 1794, S. 64. 
gliedern. Erstens ging es um allgemeine liturgische Feste, Gottesdienste und Andachten und dazu gestiftete Musik. Zweitens handelte es sich um Gottesdienste, die ausschließlich mit dem liturgischen Betrieb im Dom verbunden waren, wie Jahrestage, Gottesdienste in der Gnadenbildkapelle und bei dem alten Gnadenbild sowie die Exequien, denen vor allem nach dem Tod eines Herrschers eine besondere Bedeutung zukommt.

\section{Allgemeine liturgischen Feste}

Im Advent fanden im Dom Rorate-Messen statt. Für die Mitwirkung erhielten sowohl alle Sänger als auch die Posaunisten eine Sonderzahlung. ${ }^{36}$ Für die Rorate-Messe wurden noch zwei zusätzliche Choralisten engagiert, nämlich ein Tenorist und ein Bassist. Im Jahre 1783 wurden die Sänger und Instrumentalisten für die Rorate-Messe zum letzten Mal entlohnt. Ab dem Jahre 1785 übernahm diesen Dienst, „die Beistimmung der Orgel bey der Rorate Fruheme $\beta$ Liedern"37 der Domorganist. Diese Veränderung reduzierte die Ausgaben auf Rorate-Messen um $17 \mathrm{fl}$.

Dem Bittschreiben des Organisten Georg Summer aus dem Jahr 1809 ist zu entnehmen, dass diese Ersparnis sogar noch größer war. In der Zeitspanne von 1803 bis 1809 wurde ihm das ihm zustehende Rorategeld gar nicht gegeben, obwohl er diese Dienste leistete. Aufgrund dessen beantragte er für die genannte Zeitspanne seine Bezahlung von $48 \mathrm{fl}$. Doch das Geld wurde erst nach seinem Tod seiner Familie ausbezahlt. ${ }^{38}$

Zu Weihnachten konnten ab dem Jahr 1750 dank der Braitenbücherlichen Stiftung die Hoftrompeter „in der Weynacht bey der Metten gestüfften Musicaliscchen Ambt“"39 bis ins Jahr 1754 begleiten. Ab 1755 erhielt das gesamte Ensemble eine Bezahlung von $15 \mathrm{fl}$ für die Weihnachtsmette..$^{40}$ Im Jahre 1768 kam zur Vergütung des gesamten Ensembles 9 fl für „dazu gestiftete Trompeten und Paucken"41 hinzu.

In der Fastenzeit gab es keine speziellen Ausgaben für Musik, was mit der Besinnung des Leiden Christi zusammenhing. Wie vermutet werden kann, hatten die Musiker erst in der Karwoche verschiedene Sonderaufgaben. Am Anfang des Jahrhunderts wurden die Stadttürmer für die Prozessionsbegleitung extra besoldet, dies ist unter der Ausgabe ,für die fünf Instrumentalisten" zu finden. Diese Prozession in der Karwoche fand

36 Vgl. AT-WStLA, HS, A41/28 Kirchenmeisteramts-Rechnungen von St. Stephan - 1781, Fol. 88v.

37 AT-DAWST, KMA, Rechnungsbuch 1785, Fol. 78r.

38 AT-DAWST, KMA, Erledigungen 1808-1811, Kart. 11/1, Fol. 1453.

39 AT-WStLA, HS, A41/25 Kirchenmeisteramts-Rechnungen von St. Stephan - 1750-1751, Fol. 89v.

40 Die Angaben zur sog. Braitenbucherischen Stiftung sind in den ersten Jahren unter der Rubrik Gemeine Ausgaben, ab dem Jahre dann unter Ausgaben auf die Cantorey zu finden, ab dem Jahre 1781 kam es zuerst unter Stiftungen. Ab 1766 wurde der Betrag zwar auf 12 fl. reduziert. Vgl. AT-DAWST, KMA, Rechnungsbuch 1766, Fol. 132r.

41 AT-DAWST, KMA, Rechnungsbuch 1768, Fol. 94v. 
bis ins Jahr 1770 am Karfreitag statt. ${ }^{42}$ Ab dem Jahr 1781 wurde die Prozession dann regelmäßig - auch nach der Musikreform ${ }^{43}$ - am Gründonnerstag veranstaltet. Mit dem Triduum sind Tenebrae verbunden. Zum ersten Mal im Jahre 1755 wurde dem Kapellmeister Reutter laut des Dekrets vom 25. Juni 1754 für die Musik bei den drei Pumpermetten, bzw. Karmetten 72 fl ausbezahlt. ${ }^{44} \mathrm{Zu}$ den jährlich veranstalteten Tenebrae wurden im Jahre 1764 neue Musikalien besorgt. ${ }^{45}$ Zur Karwoche gehörte gewöhnlich der Bau des Heiligen Grabes, welcher im Stephansdom bereits für das Mittelalter belegt ist. Im 18. Jahrhundert wurde für seinen Auf- und Abbau ein Tischler beauftragt. ${ }^{46}$ Regelmäßig kommen Zahlungen für die Befüllung der Lampen in der Karfreitagsnacht vor sowie an die Polizei für die gehaltene Wache, da die ganze Nacht hindurch die Kirche geöffnet war. Ansonsten gibt es keine Hinweise auf Oratorienaufführungen oder andere Musikdarbietungen beim Heiligen Grab.

Weitere große Feierlichkeiten, für die die Hoftrompeter und ein Pauker bis ins Jahr 1754 engagiert waren, stellte die Auferstehungsfeier dar. ${ }^{47}$ Ab dem Jahr 1755 wurde diese Gebühr nicht mehr ausgezahlt, was vielleicht mit der Verordnung über die Verwendung der Trompeten und Pauken in der Kirche und bei den Prozessionen zusammenhing. Bemerkenswert ist, dass dies allerdings keinen Einfluss auf die Bezahlung von $3 \mathrm{fl}$ für die fünf Instrumentalisten (Posaunisten) bei der Karfreitagsprozession hatte. Die Gebühr (gekürzt auf $7 \mathrm{fl}$ ) für Hoftrompeter und Pauker für die Mitwirkung beim Hochamt am heiligen Ostersonntag tauchte wieder im Jahre 1770 unter den Ausgaben der Gnadenbildkapelle auf. ${ }^{48}$ Für die feierliche Messe am Pfingstsonntag wurde das Ensemble durch Trompeten und Pauken ergänzt. Belegt wird dies erst ab dem Jahre 1770, wobei die Bezahlung zu Pfingsten mit $7 \mathrm{fl}$ der Bezahlung zu Ostern entsprach. ${ }^{49}$

Die Begleitung der Fronleichnamsprozession verrichtete ab 1779 das Ensemble des Hoftrompeters, jedoch nur bis $1782 .{ }^{50}$ Aus dem Jahre 1800 stammen Belege, aus denen hervorgeht, dass bei dieser Gelegenheit regelmäßig die Landschaftstrompeter mit der Prozession mitgingen. Dafür erhielten sie jedes Mal $10 \mathrm{fl}$ und für den Paukenträger

42 Die Prozession wurde zuerst aus der Stiftungsgelder bezahlt, danach ist aber bei den Ausgaben auf die Vokal- und Instrumentalmusik erfasst.

43 Siehe Auswirkungen der Gottesdienstreform Kaiser Joseph II. und Reform der Dommusik. In KRAMÁř́VÁ, Helena. Die Dommusik zu St. Stephan im 18. Jahrhundert in sozialgeschichtlicher Perspektive. In Musicologica Brunensia, 54, 2019, Nr. 1, S. 173-195.

44 Diese Gebühr ist ab 1767 über Abzug der 4\% $14 \mathrm{fl}$ 24x auf 57 fl 36x. Vgl. AT-DAWST, KMA, Rechnungsbuch 1755, Fol. 75v; ebenda 1767, Fol. 121v.

45 Siehe Musikalien für die Essential-Kapelle.

46 Nachweislich wurde vom 1770 bis 1774 ein Tischler mit für 4 fl. entlohnt. Vgl. AT-DAWST, KMA, Rechnungsbuch 1774, Fol. 68v.

$47 \mathrm{Ab}$ dem Jahre 1722 erhielte „wirdet dieses zum ersten mahl wegen verrichtenden dienst bey der auferstehung Unseres Lieben Herrn auf Verordnung Nr. 331 und Quittung Nr. 332 behalt mit 12 fl., welche Gebühr Vermög angezogener gnädigen Verordnung hinführo Jährlich bezahlt zu werden continuiert werden soll." AT-DAWST, KMA, Rechnungsbuch 1722, Fol. 61r.

48 AT-DAWST, KMA, Rechnungsbuch 1770, Fol. 86r.

49 Ebenda, Fol. 86r.

50 AT-DAWST, KMA, Rechnungsbuch 1782, Fol. 92r. 
$1 \mathrm{fl}$, ab wann wird nicht angegeben. Es lässt sich jedoch annehmen, dass sie von den Hoftrompetern diese Tätigkeit ab 1783 übernommen haben könnten. Im April 1800 richteten sie aber dem Magistrat aus, dass sie diese Dienste für diesen Preis nicht mehr leisten könnten und forderten ab sofort für jede Teilnahme $15 \mathrm{fl}$ für die Musiker und wie zuvor $1 \mathrm{fl}$ für den Paukenträger. Laut des zuständigen Kirchenmeisters war die geforderte Erhöhung einerseits zwar sehr groß, anderseits mangelte es aber an fähigen Musikern. Er behauptete, dass es in ganz Wien nicht mehr als drei Trompeterensembles gäbe und es daher für die Domkirche schwer werden würde, einen ordentlichen Ersatz zu finden. Aus diesen Gründen wurde die Preiserhöhung am 26. April 1800 genehmigt. ${ }^{51}$

Unter die hohen Feste wurde auch das Fest des heiligen Leopold, der sogenannte Leopoldi-Tag gezählt. Den Rechnungen nach erfolgte eine Sonderzahlung vom $4 \mathrm{fl}$ für zwei Clarinen und Pauken an den Hoftrompeter Peter Hofbauer ebenfalls nur von 1779 bis ins Jahr 1783. Ab dem Jahre 1784 wurden die "gemachten Dienste" der Hoftrompeter nicht ausführlich beschrieben, deshalb lässt sich nicht bestimmen, bei welchen Gelegenheiten sie mitwirkten.

\section{Andachten}

In der Domkirche fanden regelmäßig verschiedene Andachten statt, eine der wichtigsten davon war das Vierzigstündige Gebet. ${ }^{52}$ Im Jahre 1721 fand es seinen Abschluss am 27. August im Stephansdom. Der kaiserliche Trompeter wurde „wegen des den 27. August 1721 alda abgehaltenen schluss des 40. Stündigen gebets" ${ }^{\text {53 }}$ mit $9 \mathrm{fl} 15 \mathrm{x}$ belohnt. Im Jahre 1750 wurde die Mitwirkung bei dem zweimal gehaltenen Vierzigstündigen Gebet mit $18 \mathrm{fl}$ entlohnt. ${ }^{54} \mathrm{Im}$ Jahre 1757 verfertigte der kaiserliche Hof- und Theatral-Maler Franz Anton Danne vier neue Bilder zum Vierzigstündigen Gebet für $100 \mathrm{fl}$ an. ${ }^{55}$ Weitere Nachrichten über die musikalische Umrahmung des Vierzigstündigen Gebetes stammen vom 20. Januar 1791; damals beschwerte sich der Hoftrompeter Peter Neuhold darüber, dass er mit seinen Musikern für das Mitwirken am Vierzigstündigen Gebet keine Bezahlung seit der Musikreform erhalten hätte, obschon dies nicht zu den ordentlichen Pflichten gehören würde. Peter Neuhold war der Meinung, die Trompeter sollten jedes Mal für ihre verrichteten Dienste bei dieser Gelegenheit $5 \mathrm{fl}$ erhalten.$^{56}$ Dies wurde gebilligt, obschon der Magistrat der Meinung war, dass die Musiker bei der Mitwirkung am Vierzigstündigen Gebet ,fast gar nichts zu tun hatten, und daher ganz überschüssig

51 AT-DAWST, KMA, Erledigungen 1800-1804, Kart. 9/1, Fol. 1022.

52 Vgl. HERSCHE, Peter. Muße und Verschwendung: Europäische Gesellschaft und Kultur im Barockzeitalter. Freiburg, Herder Verlag, 2006, S. 570.

53 AT-DAWST, KMA, Rechnungsbuch 1721, Fol. 54v.

54 AT-DAWST, KMA, Rechnungsbuch 1750, Fol. Fol. 81v.

55 Überdies lieferte er in diesem Jahr auch ein neugemachter Ölberg für 150 fl. Vgl. AT-DAWST, KMA, Rechnungsbuch 1757, Fol. 66r.

56 AT-DAWST, KMA, Erledigungen 1792-1800, Kart. 8/1, Fol. 737. 
waren, folglich auch die geringere Besoldung noch zu groß war. "57 Nach zehn Jahren wurde die Vergütung der Trompeter noch einmal diskutiert, da inzwischen beim Vierzigstündigen Gebet das deutsche Te Deum eingeführt worden war, bei dem ein Chor der Trompeter und Pauker (oder Chor der Trompeten und Pauken) notwendig war. Der Kirchenmeister Weißer glaubte daher, dass es besser wäre, die alte Bezahlung von $9 \mathrm{fl} \mathrm{zu}$ bewilligen, da ansonsten die Unkosten für die Kirchenkasse deutlich größer werden würden. Die HofTrompeter verlangten nämlich bei zweimaliger Abhaltung des Vierzigstündigen Gebets in der Domkirche St. Stephan sowie in der Kirche St. Salvator pro Gotteshaus $8 \mathrm{fl.} .^{58}$

Eine besondere Stellung hatte im Dom die Marianische Verehrung. An den sechs größten Marianischen Festtagen wurden bei dem sog. Alten Gnadenbild - gemeint ist das Bildnis „Maria in der Sonne“ - von Essential-Vokalisten bis ins Jahr 1784 Litaneien in Choralbesetzung gesungen. ${ }^{59}$ Außer diesen gestifteten Litaneien beim alten Gnadenbild wurde das gesamte Ensemble ,für die verrichteten Musikalischen Ämter; und Litaneyen an großen Fest [!]; und Frauen Tägen "60 vergütet. An den Frauentagen wurde auch der Domorganist „wegen Schlagung des Lieds"61 mit $6 \mathrm{fl}$ belohnt.

Am Ende des 18. Jahrhunderts wurden in der Domkirche Kriegsandachten analog zu anderen Kirchen gehalten. ${ }^{62}$ Jeden Sonntag von 10 bis 11 Uhr gab es eine solche Andacht im Dom, bei deren erstem und letztem Segen die Orgel gespielt werden musste. Diese Dienste verrichtete der Organisten-Adjunkt Georg Summer; er wurde dafür nicht bonifiziert, obwohl es in den anderen Kirchen üblich war. Seine Bitte um eine Remuneration wurde mit einer einmaligen Zahlung von $50 \mathrm{fl}$ ohne Anspruch auf weitere Zuschüsse abgegolten. ${ }^{63}$

\section{Besondere Gottesdienste}

Der regelmäßig verrichtete Musikbetrieb wurde im Laufe des 18. Jahrhunderts (bis 1784) nur selten verändert, trotzdem gab es einige Gelegenheiten, die für das Musikensemble zusätzliche Dienste bedeutete. Gesprochen ist über mit der barocken Mentalität und Religiosität verbundenen Feierlichkeiten und verschiedenen Andachten im Dom. Die wichtigsten Ereignisse davon sind mit der Verehrung des Gnadenbilds Mariä Pötsch verbunden, nicht weniger von Bedeutung waren die Exequien nach dem Tod eines Herr-

\footnotetext{
57 AT-DAWST, KMA, Erledigungen 1800-1804, Kart. 9/1, Fol. 1068.

58 Es handelte sich um die Andachten bei St. Stephan und St. Salvator. Vgl. Ibidem, Fol. 1068.

59 Es ging um folgende sechs Marianische Feste: 2. Februar - Mariä Purificatio (Maria Lichtmesse) 25. März Annuntiatio Domini (Verkündigung des Herrn), 2. Juli - Visitatio Mariae (Maria Heimsuchung), 15. August - Assumptio Beatae Mariae Virginis (Mariä Himmelfahrt), 8. September - Mariä Geburt, 8. Dezember - Immaculata conceptio (Unbefleckte Empfängnis).

60 AT-DAWST, KMA, Rechnungsbuch 1783, Fol. 103v.

61 AT-DAWST, KMA, Rechnungsbuch 1784, Fol. 121r.

62 AT-DAWST, KMA, Erledigungen 1800-1804, Kart. 9/1, Fol. 1047.

63 AT-DAWST, KMA, Erledigungen 1800-1804, Kart. 9/1, Fol. 1051.
} 
schers, zusätzlich wurden die Musiker auch wegen anderer Andachten besoldet. An dieser Stelle werden die Feste angegangen, die ausschließlich mit dem liturgischen Betrieb im Stephansdom verbunden waren.

\section{Gottesdienste zur Verehrung des Gnadenbildes Maria Pötsch}

Wie bereits angedeutet, erfreute sich die Mutter Gottes außerordentlicher Wertschätzung. Diese zeigte sich vor allem bei dem Gnadenbild Maria Pötsch, bei dem ein eigener täglicher liturgischer Betrieb mittels Stiftungen gewährleistet wurde. Die Unkosten für den liturgischen Betrieb bei diesem Altar, handwerkliche Arbeiten und Einkäufe, sowie die Musikkosten deckte der Wiener Magistrat.

Das liturgische Geschehen am Altar Maria Pötsch umfasste mehrere gelesene Gottesdienste zwischen 5 Uhr morgens und mittags, darunter ein zelebriertes Hochamt um 11 Uhr, nachmittags dann um 17 Uhr gehaltene Litaneien. Dazu kam dreimal täglich das Rosenkranzgebet (eine halbe Stunde vor der Messe um 9 und 12 Uhr und nach den Litaneien). Anzunehmen ist, dass die Gnadenbildkapelle das Hochamt und die Litaneien musikalisch gestaltete. ${ }^{64}$ Die Musikkosten von $1702^{65}$ bis in das Jahr 1783 bewegten sich jährlich zwischen $2.262 \mathrm{fl}$ und $2.986 \mathrm{fl}$; Bis ins Jahr 1753 beispielsweise waren davon $2.058 \mathrm{fl}$ für das gesamte Ensemble und $600 \mathrm{fl}$ für die Hoftrompeter bestimmt. ${ }^{66}$

Die Gedenktage des Tränenwunders und die Überstellung des Marianischen Bildnisses von Ungarn nach Wien wurden besonders gefeiert. ${ }^{67}$ Ein besonderes Ereignis war ein Jubelfest zum fünfzigjährigen Jubiläum des wundertätigen Gnadenbilds Maria Pötsch, das im Jahre 1747 stattfand. Die achttägige Festivität wurde laut des dazu herausgegebenen Druckes nicht nur in der Metropolitan-Kirche zu St. Stephan mit verschiedenen Trostpredigten, Hochämtern und Litaneien vom 1. bis 9. Juli begangen, sondern auch in anderen Wiener Kirchen. ${ }^{68}$ Die heutzutage vorhandenen Quellen lassen nur eine sehr beschränkte Einsicht in die damaligen Feierlichkeiten zu, veranschaulichen jedoch zumindest die spätbarocke Religiosität, die durch viele andere Festivitäten bereits belegt ist. ${ }^{69}$

Die Domkirche wurde mithilfe unterschiedlicher Mittel geschmückt - zu erwähnen ist Niederländischer Spalier, grüne Bäume und verschiedene Verzierungen von einem

64 Mit der Musikpflege am Altar Maria Pötsch beschäftigten sich zum ersten Mal ausführlicher: HOCHRADNER - VÖSÖMARTY, op. cit., S. 133-175.

65 In dem Aufsatz von HOCHRADNER-VÖSÖMARTY beginnen die Kirchenrechnungen erst mit dem Jahr 1709, das Rechnungsbuch aus dem Jahre 1702 wird im Wiener Stadt- und Landesarchiv aufbewahrt und wurde in ihrem Aufsatz nicht berücksichtigt.

66 Für jährliche summarische Ausgaben der Gnadenbildkapelle auf die Musik siehe die tabellarische Darstellung In HOCHRADNER - VÖSÖSMARTY, op. cit., S. 156-164.

67 Siehe: HOCHRADNER - VÖSÖSMARTY, op. cit., S. 139-142.

68 Anlässlich dieser Gelegenheit ist ein Druck herausgegeben: Funftzig-Jähriges Jubel-Fest des wunderthätigen Gnaden-Bilds Mariae von Pötsch [...] Wien, 1747 (ÖNB 74.632-B).

69 Zu Funktionen der Festivitäten und deren Rolle in der spätbarocken Gesellschaft siehe: HERSCHE, Peter. Muße und Verschwendung: Europäische Gesellschaft und Kultur im Barockzeitalter. Freiburg, Herder Verlag, 2006. 
bürgerlichen Cranzlbindler. Zwecks der Festlichkeiten wurde ebenfalls ein neuer Baldachin aus rotem Samt, sowie ein „Christallen-Hängeleuchter" besorgt. Die von Künstlern gelieferten Arbeiten wurde leider nicht genauer spezifiziert; auf jedem Fall bekam der Bildhauer Georg Hillebrand für verrichtete Arbeit 18 fl; der Theatral-Maler Franz Anton Danne verlangte dagegen $223 \mathrm{fl}$, was beispielsweise eine Abgeltung sein könnte für die künstlerische Gestaltung eines Ehrengerüstes oder für die Herstellung temporärer thematischer Prospektbilder, die in der Kirche aufgehängt werden konnten.

Die Angaben betreffend die Musik sind ähnlich knapp formuliert - trotzdem deuten die Quellen in der Summe auf ein umfangreiches musikalisches Programm während des halbrunden Jubiläums hin. Den Hoftrompetern wurden laut der Ratsverordnung zuerst 63 fl zuerkannt, dazu kam noch eine extra Zahlung von $27 \mathrm{fl} 30 \mathrm{x}$, somit zusammen $90 \mathrm{fl} 30$ x. Zu vermuten wäre, dass die Musiker der Leitung des Gnadenbildkapellmeisters Ferdinand Schmid unterstanden. Während des Festes hatte jedoch der Domkapellmeister Georg von Reutter d. Ä. die Leitung der Musiker inne; Sämtliche Musiker wurden mit $150 \mathrm{fl}$ besoldet, hinzu kamen weitere $30 \mathrm{fl}$. für die extra Musicos, die für eine Verstärkung bzw. Besetzungsvergrößerung sorgten. ${ }^{70}$ Daher ist anzunehmen, dass nicht nur die Essentialisten, sondern auch die Musiker der Gnadenbildkapelle, möglicherweise aber auch noch zusätzlich eingeladene Musiker bei dieser Festivität mitwirkten. Für diese Festivitäten durften neue Musikalien besorgt werden, obwohl das Fest leider bei keinem der Einträge in den Rechnungsbüchern ausdrücklich vermerkt wurde.

\section{Jahrestage, Requiem und Libera}

Unter zahlreichen gehaltenen Gottesdiensten an gestifteten Jahrestagen inkludierten nur die wenigsten das Geld für spezielle Musikbegleitung. In den meisten Fällen wurde für das Musizieren an den Jahrestagen das ganze Essentialkapelle entlohnt; die Bescheinigungen wurden immer vom jeweiligen Kapellmeister erstellt. Alljährlich wurden in Rahmen der sog. Quatember-Tage (von lat. ieiunia quattuor temporum „Fasten der vier [Jahres]zeiten“) vier Requiem musikalisch gestaltet. Die Dommusik wurde von der sog. Kirchenmeisterlichen Quatenberlichen Stiftung finanziert. Dazu kam im Jahre 1717 ein musikalisches Requiem für $10 \mathrm{fl}$ aus der Huttauerische Stiftung am Sterbetag von Andreas Huttauer. Im Vergleich zu den vorherigen wurde für die Musik am Gedenktag des Todes von Fürsten und Kardinal Trautson deutlich aufwändiger gestaltet; für das Requiem zusammen mit dem anknüpfenden Libera me wurde $24 \mathrm{fl} \mathrm{regelmäßig} \mathrm{bezahlt.}{ }^{71}$

70 Diese Einträge befinden sich zusammen mit den anderen Ausgaben bezüglich der Festivität in der Rubrik „Auf Handl- und Handwerckersleuth“. Vgl. AT-DAWST, KMA, Rechnungsbuch 1747, Fol. 61v-63r.

71 AT-DAWST, KMA, Rechnungsbuch 1758, Fol. 48v. 


\section{Exequien}

Wie zuvor angedeutet, nahmen die für einen verstorbenen Herrscher gehaltenen Exequien eine Sonderstellung ein. Obzwar dies nicht so häufig vorkam, stellten sie trotzdem eine wichtige Angelegenheit dar. Zwischen dem 22. und 24. März 1741 fanden in St. Stephan Exequien für den verstorbenen Kaiser Karl VI. ${ }^{72}$ statt, was die publizierte Leichenpredigt und der Kupferstich des von Franz Anton Danne entworfenen Trauergerüsts belegt. ${ }^{73}$ Da der Jahrgang 1741 der Rechnungsbücher nicht zur Verfügung steht, kann nur anhand der später veranstalteten Exequien behauptet werden, dass die Dommusik dabei eine bedeutende Rolle gespielt haben muss. 1765 empfing das Oberkammeramt $476 \mathrm{fl} 46$ 1/2 x, um für den verstorbenen Franz I. Stephan von Lothringen dreitägige Exequien im Stephansdom vorzubereiten. Von diesem Geld bekamen die Musikensembles $120 \mathrm{fl}$ für ihre Mitwirkung bei vier Ämtern. ${ }^{74}$

Am 29., 30. und 31. Januar 1781 wurden dreitägige Exequien für die verstorbene Maria Theresia in der Metropolitankirche zu St. Stephan gehalten. Inmitten der Kirche wurde ein Trauergerüst (Castrum doloris) aufgerichtet, das während der Feierlichkeiten prächtig beleuchtet wurde. Jeden Tag wurden nach der Trauerrede des Dompredigers Joseph Schneller Nokturnen des Totenoffiziums vom Domkapitel und sämtlichen Kurgeistlichen gesungen und danach das musikalische hohe Seelenamt gehalten. Im Anschluss daran ging ein Umzug zu dem Trauergerüst, dort wurden die Responsorien gesungen und ein feierlicher Segen erteilt. Der Magistrat übernahm fast alle Unkosten inkl. der Hälfte der Wachskosten (449 fl 29 x). ${ }^{75}$ Die andere Hälfte sowie die Musiker wurden aus der Kirchenkasse vergütet. Für ein dreimal gehaltenes musikalisches solennes Requiem und Libera verlangte der Kapellmeister Leopold Hofmann 102 fl 24 x für die ganze Musik. ${ }^{76}$

Als Kaiser Joseph II. starb, sind keine Ausgaben zu Exequien zu finden, da die Rechnungsbücher für das betreffende Jahr fehlen. Nach dem Tod seines Nachfolgers Leopold II. wurde für das Castrum doloris und andere Unkosten, die ebenfalls vom Oberkammeramt gestiftet wurden, insgesamt $1.497 \mathrm{fl} 28 \mathrm{x}$ ausgegeben,, wovon $108 \mathrm{fl}$ für die Musik bestimmt waren. Im gleichen Jahr wurden ebenfalls für seine verstorbene Gattin Exequien gehalten, wobei die Musik für alle drei Tage nur 57 fl 36 x erhielt. ${ }^{77}$

72 Vgl. BRIX, Michael. Trauergerüste für die Habsburger in Wien. In Wiener Jahrbuch Für Kunstgeschichte, 26(1), 1973, S. 265.

73 Vgl. SOMMER-MATHIS, Andrea - FRANKE, Daniela - RISATTI, Rudi. Spectacolo Barocco! Triumph des Theaters, Wien: Michael Imhof Verlag, 2017, S. 327.

74 AT-DAWST, KMA, Rechnungsbuch 1765, Fol. 154r.

75 AT-WStLA, HS, A41/28 Kirchenmeisteramts-Rechnungen von St. Stephan - 1781, Fol. 39r.

76 AT-WStLA, HS A41/28 Kirchenmeisteramts-Rechnungen von St. Stephan - 1781, Fol. 91r.

77 Die Gesamtkosten betrugen in diesem Fall nur 235 fl 21x.Vgl. AT-DAWST, KMA, Rechnungsbuch 1792, S. 68 . 


\section{Finanzierungsquellen der Dommusik}

Die Unkosten für das oben geschilderte, aufwändige Musikgeschehen wurden aus mehreren Geldquellen gedeckt. Die meisten Kosten wurden aus der Kirchenkasse beglichen, zweitwichtig waren Erträge aus den Musikstiftungen. Nicht zu vergessen ist die bei der Domkirche aktive Bruderschaft Corpus Christi. Im Folgenden wird auf die zwei Letztgenannten näher eingegangen.

Die Musikstiftungen trugen auf zwei möglichen Wegen zur Verschönerung des liturgischen Betriebs bei. Entweder konnten aus ihren Mitteln allgemeine liturgische Feste finanziell gefördert und die musikalische Begleitung bei wichtigen Ereignissen des Kirchenjahres finanziell unterstützt werden; oder es wurde durch das angelegte Kapital der regelmäßige, jedoch nicht allgemein verbindliche Betrieb gesichert. Bei St. Stephan handelte es sich beispielsweise um die Gottesdienste an den Jahrestagen; bei einigen von ihnen wurde eine musikalische Beteiligung erwünscht. Durch die Stiftungen wurde es den im Stephansdom regulär angestellten Sängern und Musikern ermöglicht, durch diese zusätzlichen Dienste ihre finanziellen Verhältnisse zu verbessern.

Die Musiker wurden von mehreren Stiftungen bezahlt. Aus den ältesten Quellen geht hervor, dass bereits am Ende des 17. Jahrhunderts an den sechs Frauentagen beim alten Gnadenbild Choral-Litaneien gehalten wurden, die aus Stiftungsgeldern bezahlt worden waren. Dem Kapellmeister wurden jährlich $36 \mathrm{fl}$ bezahlt. Diese Zahlung erfolgte jährlich bis ins Jahr 1762 unverändert. Ab diesem Jahr wurde der Betrag unter der angegebenen Choralistenbesoldung als "Chörl-Litaney“ erfasst. ${ }^{78}$ Im Jahre 1766 kam es bei den einzelnen Zahlungen zu Abzügen, sodass sich der Gesamtbetrag von $36 \mathrm{fl}$ auf $28 \mathrm{fl} 48 \mathrm{x}$ reduzierte. So blieb es bis ins Jahr 1781, in welchem der zweite Bassist Tobias Gsur die Zahlung für die getätigten Chor-Litaneien für alle neun Choralisten über $33 \mathrm{fl} 36$ x quittierte. Er übernahm die Bezahlung zum letzten Mal im Jahre 1783, danach wurden die Litaneien nicht mehr bezahlt, da sie nach der Musikreform höchstwahrscheinlich nicht mehr stattfinden konnten.

Sehr ähnlich scheint der Verlauf und das Schicksal der sog. Kirchenmeisterlichen Quatenberglichen Stiftung zu sein. Aus dieser Stiftung wurden dem Kapellmeister und dem gesamten Ensemble für vier gehaltene Requien während der Quatember-Tage mit $26 \mathrm{fl}$ bezahlt. So wie bei der vorherigen Stiftung verschwand diese Ausgabe aus der Rubrik „Auf die gestiffteten Messen, Litaneyen, Angst und Requiem“ im Jahre 1762 und ist seitdem unter einzelnen Choralisten-Quittungen zu finden, jedoch unter der Bezeichnung Kirchenmeisterliches Requiem ohne weitere Angaben. Zunächst blieb die Höhe der Auszahlung von $26 \mathrm{fl}$ gleich, später kam es zum Abzug ${ }^{79}$ von $2 \mathrm{fl}$ pro Zahlung. Damit erhielten die Choralisten also ab dem Jahr 1765 oder 1766 insgesamt 18 fl für vier gehaltene Requien aus dieser Stiftung. Zwischen den Jahren 1781 und 1783 wurden Tobias Gsur nur

78 Vgl. AT-DAWST, KMA, Rechnungsbuch 1762, Fol. 97v-99r.

79 Dieser Abzug bei einigen Erträgen von Stiftungsgelder, aber auch bei der Bezahlung der Pumpermetten in der Karwoche hängt mit Steuerverordnungen und -regulation in den 60er Jahren zusammen. 
$16 \mathrm{fl}$ gegeben. Sowohl die gestifteten Chorallitaneien als auch das Kirchenmeisterliche Requiem sind im Laufe der Zeit zum festen Bestandteil der angestellten Sänger in der Domkirchen geworden und zählten ab den 1760er Jahren zu den regulären Pflichten der Choralisten.

Während des 18. Jahrhunderts wurden weitere Stiftungen gegründet. Im Jahre 1707 ließ der Handelsmann Michael Kurz in seinem Testament das Kapital von $3.100 \mathrm{fl}$ für die Musik im Dom anlegen. Die Erträge der Kurzischen Stiftung sollten für die Bezahlung der großbesetzten Musik mit Trompeten und Pauken für die Gottesdienste beim Gnadenbild an allen Sonn- und Feiertagen dienen. ${ }^{80}$ In den 1760er Jahren konnte aus dem Stiftungsüberschuss sogar die Musik für beide Ämter der Bruderschaft Corporis Christi finanziell unterstützt werden. So betrug beispielsweise der Ertrag der Stiftung im Jahre $1781124 \mathrm{fl}$.

Ebenso stellten die Erträge der Vestenburglichen Stiftung eine weitere bedeutende Quelle für die Musik in der St. Stephanskirche dar. Im Jahre 1739 wurden von Franz Leopold Vestenburg 4.900 fl gestiftet. ${ }^{81}$ Aus diesen Erträgen erhielten die musikalischen Ensembles jährlich 230 fl. Diese Förderung diente der Vergrößerung der Essentialkapelle bei den Hochämtern und Litaneien am großen Fest und an Frauen-Tagen (die oben erwähnten Marianischen Feste). Im Jahre 1781 betrug der Ertrag 196 fl, ${ }^{82}$ wovon $184 \mathrm{fl}$ für die Musik verwendet wurden.

Im Jahre 1717 kam zu den Stiftungen die sog. Huttauerische Stiftung hinzu; das gesamte Musikensemble erhielt für das Mitwirken beim gehaltenen Jahrestag-Gottesdienst bzw. für das musikalische Requiem $10 \mathrm{fl}$ (ab $17668 \mathrm{fl}$ ) aus dem Gesamtgewinn von $102 \mathrm{fl}$. Das von Andreas Hüttauer angelegte Kapital betrug 2.550 fl. ${ }^{83}$ Die nach dem 1749 verstorbenen Weihbischof Joseph Heinrich von Braitenbucher benannte Braitenbücherliche Stiftung wurde mit einem Kapital von $600 \mathrm{fl}$ ausgestattet. Der jährliche Ertrag betrug 24 $\mathrm{fl}^{84}$, woraus $13 \mathrm{fl} 30 \mathrm{x}$ für die Hoftrompeter für die Mitwirkung bei der Weihnachtsmette bestimmt wurden. Zu den weiteren Jahrestagen, bei denen das gesamte Ensemble mitwirkte, gehörte der Fürst und Kardinal Trautsonsche Jahrestag. Nach dem Tod des Fürstbischofs und Kardinals Johann Joseph von Trautson am 10. März 1757 wurden - wie testamentarisch verfügt $-4.000 \mathrm{fl}$ angelegt. ${ }^{85}$. Anders als bei allen vorher erwähnten Stiftungen wurden den Musikensembles aus der Trautsonschen Stiftung nach der Musikreform noch $19 \mathrm{fl} 12$ x für die musikalische Gestaltung des Jahrestages ausbezahlt. ${ }^{86}$

80 AT-WStLA, Hauptarchiv, A1 Akten und Verträge 9/1707, 27. August 1707, Testament des hofbefreiten Handelsmannes Michael Kurz (Widmung für Gnadenbild Unser Lieben Frau zu Sankt Stephan).

81 Nachweisbar zum ersten Mal für das Jahr 1742. Vgl. AT-WStLA, HS, A41/24 Kirchenmeisteramts-Rechnungen von St. Stephan - 1742, Fol. 83v-84r.

82 AT-DAWST, KMA, Rechnungsbuch 1781, Fol. 16v.

83 AT-WStLA, Stadtarchiv, Historische Registratur, A6/2 Messstiftungen: 2.14/1 Andre Hüttauer (17181772), 2.14/2 Andre Hüttauer (1718-1742).

84 AT-WStLA, HS, A41/28 Kirchenmeisteramts-Rechnungen von St. Stephan - 1781, Fol. 10.

85 AT-DAWST, KMA, Rechnungsbuch 1758, Fol. 48v.

86 In den Rechnungen aus dem 18. Jahrhundert ist die letzte belegte Vergütung der Dommusik im Jahre 1794. Dabei kann vermutet werden, dass die Musiker weiterhin für das Requiem und Libera entlohnt wurden. Vgl. AT-DAWST, KMA, Rechnungsbuch 1794, S. 52. 
Im Gegensatz zu den Stiftungen war die Finanzierung der Musik durch die Bruderschaften im 18. Jahrhundert geringer. Die Ensembles wurden nur durch die Mittel der Corporis-Christi-Bruderschaft unterstützt. Unter der Rubrik Gemeiner Empfang wurden insgesamt $150 \mathrm{fl}$ jährlich in Quartalsraten an die Vokalisten ausbezahlt. ${ }^{87}$ Darüber hinaus beteiligte sich die Bruderschaft auch manchmal an den Kosten für Musikinstrumente.

\section{Instrumentarium}

Die Dommusik verfügte über eigene Musikinstrumente - sowohl Streich- als auch Blasinstrumente. Erste Anhaltspunkte über Menge und Zustand der Instrumente geben die Inventarien des Kircheneigentums. Zusätzliche Informationen ergeben sich aus den einzelnen Einträgen der Rechnungsbücher und weiterem Aktenmaterial; berichtet wurde hauptsächlich über Reparaturen und Anschaffungen neuer Instrumente sowie Kostenvorschläge oder Einkaufsbewilligungen.

\section{Verwendete Orgeln - Anschaffung, Reparatur, Umbauten und Pflege ${ }^{88}$}

Die Kirchenmusik ist ohne Orgel kaum vorstellbar; die täglich verwendeten Orgeln benötigten eine regelmäßige Pflege. Im Stephansdom wurde dies mit dem Begriff Bestallung verbunden. Der Bestallungsbetrag inkludierte die Orgelpflege und die Stimmung der Orgeln; dafür wurden ein oder mehrere Orgelmacher bezahlt. Zunächst erhielten zwei Orgelmacher jeweils 18 fl, pro Jahr, später nur noch ein Orgelmacher jährlich 36 fl. Ab dem Jahre 1731 erhöhte sich der Betrag auf 50 fl, was wahrscheinlich mit der VergröBerung der Orgel oberhalb der unteren Sakristei zusammenhing oder dem Wunsch, die Orgeln öfters zu stimmen. Die Orgelbestallung wurde im Jahre 1788 zum Thema - der Domorganist Matthäus Mittelmayer schickte dem Orgelmacher Franz Christoph eine Nachricht, dass er die Orgel stimmen solle, weil es seine Pflicht im Rahmen der Bestallung sei. Dieser antwortete, dass er dies unter diesen Bestallungsumständen nicht mehr machen werde und es soll die Orgel stimmen, wer wolle. ${ }^{89}$

Welche Umstände Franz Christoph meinte, ist leider aus der Korrespondenz nicht ersichtlich. Auf jeden Fall war seitdem laut Magistratsbeschluss Johann Wimola für die Bestallung der Orgeln zuständig. Johann Wimola verpflichtete sich für sechs Jahre, die zum täglichen Gebrauch bestimmte Orgel auf dem Musikchor sowie die Orgel ober-

87 AT-WStLA, HS, A41/28, Kirchenmeisteramts-Rechnungen von St. Stephan - 1781, Fol. 40v.

88 An dieser Stelle werden vor allem diejenige Informationen präsentiert, die aus den Rechnungsbüchern und erhaltenen Korrespondenz entnommen werden, um die Aussagekraft der Quellen zu präsentieren. Gründlich und ausführlicher sind Orgeln und Orgelbau in der Domkirche zu St. Stephan von Gottfired Allmer behandelt. Siehe: ALLMER, op. cit., S. 32-67.

89 AT-DAWST, KMA, Erledigungen 1786-1792, Kart. 7a/1, Fol. 609. 
halb der unteren Sakristei (auf dem Füchsel-Baldachin) zu warten und in einem guten Zustand zu halten. Voraussetzung dafür war seines Erachtens jedoch, beide Orgeln zunächst komplett zu zerlegen und die notwendigen Reparaturen durchzuführen. Für die Orgelbestallung erhielt er dann analog zu seinem Vorgänger $50 \mathrm{fl} \mathrm{jährlich.}{ }^{90}$

Dies führt uns zu der Frage, wie viele Orgeln in der Domkirche Verwendung fanden und wo sie sich befanden. Zuerst müssen Positive erwähnt werden; im Jahre 1746 wurde ein Positiv von Johann Hencke ${ }^{91}$ für die Totenkapelle für $90 \mathrm{fl}$ neu erstellt. Für dieses Positiv wurden Verzierungen vom bürgerlichen Bildhauer Johann Georg Hillbrand verfertigt. ${ }^{92}$ Für die Beschaffung eines weiteren Positivs wurden 1785 dem Organisten Mittlmayer $17 \mathrm{fl} 20 \mathrm{x}$ bezahlt, ${ }^{93}$ worüber dann keine weiteren Angaben - weder die Disposition noch der Ort, wo es sich befand - bekannt sind.

Eine neue Orgel verfertigte im Jahre 1701 Ferdinand Joseph Römer, die dann zu Pfingsten 1702 geweiht wurde..$^{94}$ Im Rechnungsbuch aus diesem Jahr wurden die Ausgaben für „Verfertigung Orgelwerck auf dem neuen Musikanten-Chor ${ }^{\prime 95}$ erfasst. Eine interessante Tatsache ist, dass die Orgel von drei Parteien gestiftet wurde: ein Teil wurde von der Domkirche bezahlt, ein weiterer von der Bruderschaft Corporis Christi beim Stephansdom und der dritte Teil aus Mitteln der Gnadenbildkapelle finanziert. Die Bruderschaft spendete noch zusätzlich $45 \mathrm{fl}$ für extra geleistete Tischler- und Vergoldungsarbeit. Bereits im Jahre 1714 musste diese Orgel für $75 \mathrm{fl}$ repariert werden. ${ }^{96}$ Weitere Ausgaben betreffend diese Orgel findet man erst wieder im Jahre 1772, als der Blasbalg-Kasten auf dem Musikchor vom Tischler verändert und die Orgel von Gottfried Sonnholz für $25 \mathrm{fl}$ repariert wurde. Aufgrund des Betrags musste es sich um eine kleinere Reparatur handeln. ${ }^{97}$

Weitere, diesmal größere Reparaturen wurden im Jahre 1785 durchgeführt. Der Orgelmacher Franz Christoph stellte eine Rechnung über $600 \mathrm{fl}$ für die Reparatur und Wiederherstellung der vorderen Orgel aus. Für die Orgelfassung und andere Arbeiten erhielt im selben Jahr der Vergolder Andreas Geiger 181 fl. ${ }^{98}$ Bevor im Jahre 1788 die Orgelbestallung Johann Wimola übernahm, war es notwendig, die kleine Orgel zu zerlegen und jede einzelne Pfeife auszuputzen. Für diese Arbeit verlangte Wimola $30 \mathrm{fl}$.

Im August 1795 war eine notwendige Wartung der kleinen Orgel auf dem vorderen Chor durchzuführen. Hier liegt ein Bittschreiben des Organisten Matthäus Mittelmayer vor, mit der Begründung, weshalb die kleine Orgel gesäubert und repariert werden sollte: „Es sind schon bereits 7 bis 8 Jahre verflossen, daß die kleine Orgel, so auf dem vordern

90 Ibidem, Fol. 608.

91 Rudolf Hopfner. Hencke (Henke, Henckl, Hengel), Johann. In Oesterreichisches Musiklexikon online, Zugriff: 13.3.2019 (http://www.musiklexikon.ac.at/ml/musik_H/Hencke_Johann.xml).

92 AT-DAWST, KMA, Rechnungsbuch 1746, Fol. 52r.

93 AT-DAWST, KMA, Rechnungsbuch 1785, Fol. 78r, Vgl. ALLMER, op. cit., S. 42.

94 Vgl. SALINGER, Arthur. Aspekte zur historischen Frage der Standorte von Orgeln im Wiener Stephansdom. In Festschrift zur Weihe der Haydn-Orgel. Wien, 2009. S. 8.

95 AT-DAWST, KMA, Rechnungsbuch 1702, Fol. 92r.

96 Ohne Angabe eines Orgelmachers. Vgl. AT-DAWST, KMA, Rechnungsbuch 1714, Fol. 57v.

97 AT-DAWST, KMA, Rechnungsbuch 1772, Fol. 67v.

98 AT-DAWST, KMA, Rechnungsbuch 1785, Fol. 62r. 
Chor stehet, nicht mehr ausgebutzet worden, durch diese lange Zeit ist sie so voll Staub und Unreinigkeit geworden, daß sie bald nicht mehr wird gebraucht werden können. " ${ }^{99}$ In seinem Kostenvoranschlag für die Wartung der kleinen Orgel berücksichtigt der Orgelmacher Wimola, dass die Arbeiten aufgrund von Andachten und Gottesdiensten häufig unterbrochen sind, er seine Mitarbeiter aber auch für diese Wartezeiten bezahlten müsse. ${ }^{100}$

Die zweite verwendete und oft erwähnte Orgel stand auf dem Füchsel-Baldachin; meist wird sie als die Orgel ober der unteren Sakristei bezeichnet. Diese Orgel wurde im Jahre 1730 von Gottfried Sonnholz gründlich repariert, gestimmt und um mehrere Register erweitert. Im darauffolgenden Jahr wurde seine Arbeit fertiggestellt und der restliche Betrag für die Reparatur ausbezahlt. ${ }^{101}$ Im Jahre 1745 wurde die Orgel nicht nur wie üblich gestimmt, sondern auch neu vergoldet. ${ }^{102}$

Ein halbes Jahrhundert später im Jahre 1788 stellte Johann Wimola fest, nachdem er sich mit der Orgel oberhalb der Sakristei eingehender beschäftigt hatte, dass besonders der Orgelkasten aufgrund seines Alters sehr beschädigt war. Sein Plan, von dem er den Kirchenverwalter sowie den Magistrat zu überzeugen versuchte, sah vor, anstelle der Orgel oberhalb der unteren Sakristei die hintere große Orgel auf der Westempore zu reparieren und zu vergrößern. Einen ersten kostengünstigen Vergrößerungsvorschlag legte Wimola im März 1789 vor. Er beschrieb darin den schadhaften Zustand der Orgel auf dem Füchsel-Baldachin. Er hob hervor, dass besonders der Orgelkasten sehr schlecht und modrig sei; da er sich überdies an einem gefährlichen Ort befände, könnte es zu einem Unglück kommen. Sein Vorschlag sah vor, die Seitenorgel vom Füchsel-Baldachin mit 24 Registern zu übertragen, die ganze Struktur neu anzulegen sowie die Klaviatur, die zum damaligen Zeitpunkt nur bis ins obere $c$ ging bis ins obere $d$ zu erweitern. Gleichzeitig gab es laut Wimola genug Platz, um zu den bisherigen 24 Registern zehn neue hinzuzufügen. ${ }^{103}$ Sein Kostenvoranschlag für die Umsetzung der Orgel von oberhalb der unteren Sakristei auf die Westempore und gleichzeitige Vergrößerung belief sich auf $5.000 \mathrm{fl}$. Jedoch „sollte die Orgl nur so wie sie ober der Sakristey ist, in den grossen Kasten auf den grossen Chor eingerichtet worden ist der Negste Preis 3400 fl." Außerdem - meinte Wimola - müsste die Orgel in dem gewöhnlichen Ton intoniert und gestimmt werden, da sie bisher um einen Viertelton zu hoch gestimmt war. ${ }^{104}$

Zum Vergrößerungsplan äußerte sich der Kirchenmeister Furtmoser, dass es derzeit unmöglich sei, diesen zu verwirklichen, da es die Kirchenkasse nicht zulasse. Seiner Ansicht nach war es dennoch höchst notwendig, dass neben der kleinen Orgel auf dem Musikchor noch eine andere Orgel zum Gebrauch bereitgestellt wird. Deshalb glaubte

99 AT-DAWST, KMA, Erledigungen 1792-1800, Kart. 8/2, Fol. 806.

100 Die Säuberungsarbeiten wurden vom Magistrat bewilligt und bestätigt, dass die Buchhaltung dem Orgelmacher $40 \mathrm{fl}$. auszahlen darf. Vgl. Ebenda, Fol. 806.

101 Diese Angaben vermittelt u. a. HASELBÖCK, op. cit., SALINGER, op. cit., ALLMER, op. cit., etc. Vgl. ATDAWST, KMA, Rechnungsbuch 1730, Fol. 43v.

102 Vgl. AT-DAWST, KMA, Rechnungsbuch 1745, Fol. 81r.

103 AT-DAWST, KMA, Erledigungen 1786-1792, Kart. 7a/2, Fol. 624.

104 Vgl. Ebenda. 
er, dass es gut wäre, wenn „die Orgel ober der Sakristey zu recht gemacht würde. "105 Der Magistrat traf daraufhin am 1. April 1789 die Entscheidung und der Orgelmacher wurde beauftragt, die Orgel oberhalb der Sakristei für $1.600 \mathrm{fl} \mathrm{zu}$ reparieren.

Nachdem Wimola mit der Reparatur dieser Orgel angefangen hatte, wiederholte er bereits am 2. Mai und danach am 15. Mai 1789 seine Bitte, dass er lieber die Orgel „in die Fassung ober der Uhr, welche recht gutt und dauerhaft wäre, versetzten und vermehret würde. "106 Bei diesem Gesuch befanden sich als Anhang drei verschiedene Pläne für den Umbau. Jedoch ist keiner davon in den Akten erhalten geblieben. Der Kirchenverwalter empfahl schließlich am 25. Mai auch die Umsetzung der Orgel aus den oben genannten Gründen; dazu meinte er, dass der Kostenvoranschlag des anderen Orgelmachers Christoph $10.000 \mathrm{fl}$ vorsähe, was doppelt so viel wäre und dass ein dauerhaftes, beständiges Orgelwerk auch der Kirche zu Ehre gereiche und sich das Publikum längst ein solches wünsche. Letztendlich genehmigte der Magistrat die „Übersetzung der Orgel ober der Sakristey auf den Haupt-Chor." 107 Im Sommer 1789 begann endlich Johann Wimola mit der Ausleerung des Orgelkastens oberhalb der Sakristei und mit der Umsetzung der Pfeifen auf die Westempore.

Aus dem Aktenmaterial ergibt sich darüber hinaus, dass die im Jahre 1720 erbaute Römer-Orgel auf der Westempore durch Wimola ersetzt wurde und die Umbau der großen Orgel spätestens 1792 vollendet wurde. ${ }^{108}$ Eine brauchbare große Orgel auf der Westempore war wahrscheinlich einer der Gründe, weshalb die Figuralmusik auf diesem Hauptchor bereits in den 1790 er Jahren stattfinden sollte. ${ }^{109}$

\section{Streich- und Blasinstrumente der Domkapellen - Anschaffung, Reparaturen und Bestallung}

Regelmäßige Einkäufe sowie Reparaturen der Musikinstrumente und andere Notwendigkeiten gehen mit dem regen Musikbetrieb im Dom Hand in Hand. Meistens wurden jährlich die Saiten einem Saitenmacher oder Lauten- und Geigenmacher bezahlt. Derselbe war oft für Reparierung der Streichinstrumente verantwortlich. Aus den Ausgaben kann natürlich auch Ausstattung des Chores mit neuen Instrumenten entnommen werden.

Das Instrumentar spiegelt zum Teil die besetzten Stellen der Essentialkapelle wider. Die Inventare des Kircheneigentums zeigen mit dem Inventar von 1732 zum ersten Mal eine Übersicht über den Instrumentenbestand zum damaligen Zeitpunkt. Unter

105 AT-DAWST, KMA, Erledigungen 1786-1792, Kart. 7a/2, Fol. 624.

106 AT-DAWST, KMA, Erledigungen 1786-1792, Kart. 7a/2, Fol. 625.

107 Vgl. Ebenda.

108 Vgl. ALLMER, op. cit., S. 51.

109 Siehe: Orte des Musizierens. 
der Rubrik „Auf deren Herren Musicanten Chor"110 wurden folgende Musikinstrumente vermerkt:

\begin{tabular}{l|l}
\multicolumn{1}{c|}{ Inventar 1732 $^{111}$} & \multicolumn{1}{c}{ Inventar 1742 $^{112}$} \\
\hline Ein neuer Violon de a $[$ nn $]$ o 1714: erkhaufft worden. & Ein neuer Violon de a[nn]o 1714: erkhaufft worden. \\
Ein Gamba, a $[$ us] 1715: & Ein neues Pässetl 1718. \\
Ein neues Bassetl 1718: & 4 neue Posaunen samt Futeral \\
Zwey neue Posaunen sambt Krumpbögen und furderal & 6 neue Violin sambt Futeral a[us]1733 \\
Sechs neue Violin furdal de a $[$ nn $]$ o $1733[$ sic! $]$ einge- & 2 deto sambt Futeral zu Vestenburgisl: Stiftung \\
schafft worden & 1 neue Brätschen sambt Futeral \\
Choralbücher & 5 alte deto, ohne Futeral \\
& 2 kupferne bauken v[on] Ihro hochwl: h: quarient
\end{tabular}

Aus diesen Einträgen geht hervor, dass in den ersten Dekaden des 18. Jahrhunderts neue Instrumente gekauft worden sind. Zwischen 1732 und 1742 wurden mehrere Musikinstrumente für die Dommusik angeschafft - 1733 wurde sechs Violinen für Essential-Violinisten eingekauft; nach der Gründung der Vestenburglichen Stiftung kamen zwei weitere Violinen für aus Stiftungsmitteln bezahlte Geiger hinzu. Im Jahre 1736 wurden vier neue Posaunen notwendig, zwei davon lieferte der bürgerliche Trompetenmacher Johann Leichnambschneider für 40 fl, zwei Futterale dazu stellte aber der Lautenmacher Anton Potsch her. ${ }^{113}$ Neue kupferne Pauken stiftete ein Mitglied der Adelsfamilien von Quarient. Überdies findet sich in den Einträgen eine Information über fünf alte Bratschen, die vorher nicht genannt wurden. In den 40er Jahren lieferte Christoph Leidolff für die Dommusik ein neues Violon. ${ }^{114}$ Es wurde wahrscheinlich aus den Mitteln der Bruderschaft Corporis Christi bezahlt und deshalb als ihr Eigentum bezeichnet. 1748 wurden dem Fagottisten Jacob Payer 16 fl 48 x für ein neues Fagott mit dem Vermerk ausbezahlt, dass es künftig bei der Kirche bleiben soll. ${ }^{115}$

In den 50er Jahren kaufte der Kapellmeister Georg Reutter d. Ä. für $10 \mathrm{fl} 47$ x nur eine neue Bratsche. ${ }^{116}$ Weitere Einkäufe fanden in 60er Jahren statt. Nach mehr als 50 Jahren wurde ein neues Violon besorgt. ${ }^{117}$ Im Jahre 1765 bekam die Witwe von Christoph Leidolff für zwei neue Bratschen 20 fl 30x. Ein Jahr später wurde es nötig, nicht nur die Streichinstrumente, sondern auch die Posaunen auszubessern, was ebenfalls seine Werk-

110 AT-WStLA, HS, A/42/2 Inventar von St. Stephan 1732, Fol. 118r.

111 Ebenda, Fol. 118r.

112 AT-WStLA, HS, A/42/3 Inventar von St. Stephan 1742, Fol. 95r.

113 Vgl. AT-DAWST, KMA, Rechnungsbuch 1736, Fol. 58.

114 AT-DAWST, KMA, Rechnungsbuch 1747, Fol. 58r.

115 AT-DAWST, KMA, Rechnungsbuch 1748, Fol. 64v.

116 AT-DAWST, KMA, Rechnungsbuch 1757, Fol. 61r.

117 AT-DAWST, KMA, Rechnungsbuch 1768, Fol. 82r. 
statt erledigte. ${ }^{118}$ Wahrscheinlich übernahm die Werkstatt des verstorbenem Christoph Leidolff im Jahre 1770 sein Sohn Joseph, denn er lieferte der Musikkapelle in diesem Jahr ein Violoncello, welches im Inventar von 1771 jedoch noch nicht vermerkt ist. ${ }^{119}$

\begin{tabular}{|c|c|}
\hline Inventar $\mathbf{1 7 6 8}^{120}$ & Inventar $\mathbf{1 7 7 1}^{121}$ \\
\hline Ein neuer Violon de a[nn]o 1714: erkaufft worden. & Ein Violon so $a[u s] 768$ : neue angeschafft worden. \\
\hline Ein neues Pässetl de $a[n n]$ o 1718. & Ein Bassetl so a [us 769 samt den futral auch neu \\
\hline $\begin{array}{l}\text { NB 12. 7bris } 769 \text { laut Passirung ein Bassetl samt } \\
\text { futeral angeschafft worden }\end{array}$ & $\begin{array}{l}\text { Ein Bassetl so a }[\text { us }\rfloor 769 \text { samt den futral auch neu } \\
\text { angeschafft worden }\end{array}$ \\
\hline 6 Violon $[!]$ de $a[n n]$ o 1733 & zwey Bratschen zu den Vestenburgisl: Stiftung \\
\hline 2 Bratschen zu Vestenburgisl: Stiftung gehörig & 6 neue Violin de a[us] 1733 \\
\hline 2 neue Bracci de a[nn]o [1]765 & 2 neue Bracci de $a[n n]$ o $[1] 765$ \\
\hline 1 Violon der Corporis Christi Bruderschaft gehörig & 1 Violon der Corporis Christi Bruderschaft gehörig \\
\hline 1 Fagott de a $[n n]$ o 748 beygeschaffet worden & 1 Fagot so $a[$ us $] 748$ beygeschaffet worden \\
\hline 4 Posaunen samt Futerall & 4 Posaunen samt den Futeral \\
\hline 2 neue Posaunen zum Conductu & 2 neue Posaunen zum Conductu \\
\hline $\begin{array}{l}2 \text { kupferne Pauken von Ihro hochwuden H: quarient } \\
\text { [Choralbücher] }\end{array}$ & $\begin{array}{l}2 \text { kupferne bauken } v[\text { on }] \text { Ihro hochwl: h: quarient } \\
{[\text { Choralbücher }]^{122}}\end{array}$ \\
\hline
\end{tabular}

Die meisten Musikinstrumente befanden sich laut den Inventaren auf dem Musikchor. Jedoch beweist ein Bewilligungsbrief vom 21. Juli 1783, dass dies nicht der einzige Ort war; nach dem Brand in der neben dem Dom stehenden Maria-Magdalena-Kapelle im Jahre 1781 wurde es nötig, neue Musikinstrumente anzuschaffen, weil manche verbrannt waren. ${ }^{123}$ Für verschiedene neue Instrumente erhielt der Hoflauten- und Geigenmacher Michael Ignaz Stadlmann 222 fl. Ein Paar große Kirchenpauken wurden vom Kupferschmied Franz Joseph Bauer für 58 fl gekauft, dazu wurden zwei Posaunen angeschafft, die vom Trompeten- und Waldhornmacher Karl Starzer verfertigt worden waren. ${ }^{124}$ Der Magistrat wies darauf hin, dass die Instrumente ausschließlich bei den Gottesdiensten in der St. Stephanskirche zu gebrauchen sind und keineswegs in anderen Kirchen oder sogar in private Häuser auszutragen sind. Für ruinierte oder abgängige Instrumente haftet der Kapellmeister und wird künftig die entstandenen Schäden aus eigenen Mittel begleichen müssen. Bei der Übergabe der Instrumente soll überdies eine Inventur stattfinden. ${ }^{125}$

118 Dies übernahm der Waldhornmacher Leichnambschneider. Vgl. AT-DAWST, KMA, Rechnungsbuch 1766, Fol. 113v und Fol. 115v.

119 AT-DAWST, KMA, Rechnungsbuch 1770, Fol. 88r.

120 AT-DAWST, Inventar 1768, [sine Folioangabe].

121 AT-DAWST, Inventar 1771, [sine Folioangabe].

122 Die Bücher sind an dieser Stelle nicht zitiert.

123 Die Frage, ob die Musikinstrumente da üblicherweise aufbewahrt wurden oder eher vergessen, lässt sich anhand der überlieferten Akten leider nicht klären. Vgl. AT-DAWST, KMA, Erledigungen 1771-1786, Kart. 7/2, Fol. 432.

124 AT-DAWST, KMA, Rechnungsbuch 1783, Fol. 77v-78r.

125 AT-DAWST, KMA, Erledigungen 1771-1786, Kart. 7/2, Fol. 432. 
Die angekündigte Inventur wurde vom Kapellmeister Leopold Hofmann ohne Begründung abgelehnt. Deshalb war noch im Oktober 1783 nicht klar, ob die Kosten für die neu angeschafften Musikinstrumente die Kirchenkasse übernimmt oder ob sie vom Kapellmeister bezahlt werden müssen. Vom Kirchenverwalter wurde zwar empfohlen, den Kapellmeister die Instrumente bezahlen zu lassen, dennoch wurden die Musikinstrumente letztendlich aus der Kirchenkasse bezahlt. ${ }^{126}$

Eine private Verwendung der Kirchenmusikinstrumente bezeugt eine Beschwerde vom 15. Juni 1791. Der Kalkant Sebastian N. ${ }^{127}$ lieh sich aus dem Bestand des Chores vier Violinen, zwei Violen und dazu ein Bassetl aus. ${ }^{128}$ Ein paar Tage danach tauchten die entlehnten Musikinstrumente wieder auf. Der Kirchenvater schrieb dazu nur eine kurze Nachricht, die auf eine interne Regelung des Vorfalls hindeutet. Sinngemäß lautete die Nachricht, dass man die Dinge auf sich beruhen lassen wolle und es nichts mehr zu klären gäbe, da die Instrumente zurückgekommen seien.

Ein Bittschreiben vom Orgelmachermeister Johann Wimola illustriert, dass Musikinstrumente nicht nur eingekauft, sondern auch verkauft wurden. Am 24. April 1795 bat er um Bewilligung zum Verkauf eines unbrauchbaren Positivs von St. Stephan an eine arme Kirche auf dem Land für 30 fl, weil das Positiv im Dom nie verwendet wird. Laut des Organisten Mittlmayer seien drei Register viel zu wenig; noch dazu müsste man das Positiv für drei Dukaten reparieren lassen. Dies sollte dann die Landkirche durchführen lassen, die das Positiv kaufen wird. ${ }^{129}$ Es könnte sich entweder um das von Johann Hencke im Jahre 1746 verfertigte Positiv aus der Totenkapelle handeln oder um das dem Organisten Mittlmayer im Jahre 1785 bezahlte Positiv. Letzteres ist wegen der geringen Registeranzahl wahrscheinlicher.

Weitere Einkäufe von Musikinstrumenten sind im Folgenden durch Korrespondenz an den Magistrat belegt. So mangelte es z. B. nach dem Tod des zweiten Geigers Niclas Reinhard am 1. Juli 1798 an einer Geige, weil sein Sohn seine Geige als Erbschaft beanspruchte. Aufgrund dessen bat der Kapellmeister Albrechtsberger am 19. September 1798 um die Bewilligung, eine neue Violine beim Geigenmacher Thier ${ }^{130}$ bestellen zu dürfen, was erlaubt wurde. ${ }^{131}$

Am 24. Oktober 1806 wurde der Kapellmeister aufgefordert, den Zustand aller Musikinstrumente des gesamten Musikpersonals der Domkirche St. Stephan zu prüfen. ${ }^{132}$ Am 5. Dezember 1806 äußerte sich der Kapellmeister Johann Georg Albrechtsberger bzgl. diverser Reparaturen von Kirchenmusikinstrumenten - wahrscheinlich als eine Reaktion an die verordnete Zustandsüberprüfung. Notwendige Reparaturen und sowie die Reinigung aller Streichinstrumente kosteten 44 fl, was der Kapellmeister sehr mäßig

126 AT-DAWST, KMA, Erledigungen 1771-1786, Kart. 7/2, Fol. 437.

127 Sein kompletter Nachname wurde in den Briefen nicht angegeben.

128 AT-DAWST, KMA, Erledigungen 1786-1792, Kart. 7a/2, Fol. 669.

129 AT-DAWST, KMA, Erledigungen 1792-1800, Kart. 8/2, Fol. 818

130 HOPFNER, Rudolf. Thier (Thir, Thür, Dirr, Dürr), Familie. In Oesterreichisches Musiklexikon online [online], 20XX [2019-03-20] URL: 〈http://www.musiklexikon.ac.at/ml/musik_T/Thier_Familie.xml〉.

131 AT-DAWST, KMA, Erledigungen 1792-1800, Kart. 8/3, Fol. 904.

132 AT-DAWST, KMA, Magistratsverordnungen 1775-1811, Kart. 6/2, Fol. 188. 
und billig fand. Da die Reparaturen günstig waren, erlaubte er sich noch um eine neue Geige zu bitten. Albrechtsberger begründete seine Bitte damit, dass auf dem Chor von St. Stephan elf Violinen vorhanden und auch elf Violinisten angestellt seien. Somit sei bei voller Besetzung kein Instrument mehr für einen Accessisten oder allenfalls mitwirkenden Dilettanten (Laien) vorhanden. Daher wäre es gut, ein Instrument im Vorrat zu haben, da solche Situationen seinen Angaben nach bereits öfters geschehen waren. ${ }^{133}$ Auf der Überschlagsrechnung von Mathias Thier, die zu Albrechtsbergers Brief hinzugelegt wurde, findet sich mit einer anderen Tinte geschrieben der Vermerk „eine nеue Violin zu verfertigen 13 fl $30 x^{\text {“ }} .{ }^{134}$

Die erwähnten Beispiele aus dem gesamten 18. Jahrhundert dokumentieren, dass für die Dommusik nicht nur neue Musikinstrumente eingekauft worden sind, sondern belegen, dass auch regelmäßige Reparaturen notwendig waren. Die Instrumentenmenge vergrößerte sich zusammen mit der steigenden Anzahl der Musiker.

\section{Musikalienanschaffungen}

Zu den gewöhnlichen Ausgaben der Musikkapelle gehörten Kosten für die Neuanschaffung sowie für die Ausbesserung der bestehenden Musikalien. Die Musikalien wurden sowohl für die Essentialkapelle als auch für die Gnadenbildkapelle angeschafft, was auf die Existenz von wenigstens zwei verschiedenen Musiksammlungen und getrennten Repertoires hindeutet. Überdies gab es zwei Arten von Zahlungen der Musikalien - bei der ersten handelte es sich um die übliche Anschaffung von Noten, indem ein oder mehrere Kopisten bestimmte Musikalien kopierten und anhand einer Ratsbewilligung oder Beglaubigung des Kapellmeisters entlohnt wurden. Zweitens finden sich in den Rechnungsbüchern Zahlungen an die Kapellmeister für neu geschaffene Kompositionen, die diese entweder noch zu ihren Lebzeiten erhielten oder die erst nach deren Tod an die Witwe gingen. Den Einträgen nach lassen sich sowohl die Zahlungen für Kopien als auch die außerordentlichen Ausgaben gut nachvollziehen. Im Folgenden wird auf die Musikalienanschaffungen für die Essential- und die Gnadenbildkapelle näher eingegangen, im Anschluss daran wird auf die Preise für einzelne Kopien fokussiert.

\section{Musikalien für die Essentialkapelle}

Mit dem Kopieren von Noten für die Essentialkapelle wurde immer ein Kopist beauftragt, es handelte sich dabei zunächst um den Fagottisten Lambert (Leonhard) Graf, ${ }^{135}$

133 AT-DAWST, KMA, Erledigungen 1804-1808, Kart. 10/2, Fol. 1322.

134 Ibidem, Fol. 1322.

135 Lambert (Leonhard, Leonard) Graf kopierte Musikalien 1709 bis 1734 für beide Musikensemble ab. 
später um den Subcantor Adam Johann Gegenbauer. ${ }^{136}$ Dessen Nachfolger war Musikus Franz Xaver Riesch, der einer der Posaunisten gewesen sein dürfte, nach ihm übernahm diese Funktion der zweite Kornettist Jacob Nurscher. ${ }^{137}$ Die Musikalienanschaffungen wurden mit ein paar Ausnahmen alljährlich durchgeführt.

Im Jahre 1702 bekam der Kapellmeister Michael Zacher für seine Kompositionen einmalig 100 fl bezahlt. Ein Dokument von 1716 gibt Aufschluss darüber, an welchem Ort die Musikalien aufbewahrt wurden. Laut dieses Dokumentes wurde Franz Cammermayer dafür entlohnt, dass er die Musikalien im Kapellhaus für 100 fl. in Ordnung brachte. ${ }^{138} \mathrm{Im}$ darauffolgenden Jahr übernahm der Kapellmeister Reutter d. Ä. für gelieferte Kompositionen die erheblich höhere Zahlung von $100 \mathrm{fl}$ pro Quartal, ausbezahlt jeweils nach Abschluss des Quartals. ${ }^{139}$ Siebzehn Jahre später, im Jahre 1735, erhielt der gleiche Kapellmeister Georg Reutter d. Ä. die beträchtliche Summe von $732 \mathrm{fl}$ „wegen abcopierten Musicalien zeit seines Capellmeisters dienst." ${ }^{140}$ Es besteht die Möglichkeit, dass es sich dabei nicht ausschließlich um Zahlungen für die Kopiatur von Kompositionen anderer Komponisten handelt, sondern in erster Linie um seine eigenen Kompositionen, die er während seiner Tätigkeit schuf und auf diese Art und Weise der Domkirche überlassen haben könnte. ${ }^{141}$

Ein weiterer, für das Kopieren von Musikalien verantwortlicher Kopist namens Adam Gegenbauer verfertigte meist vierteljährlich seine Bescheinigungen für die Kirche über abkopierte Musikalien. Im Jahre 1753 kam es zum ersten Mal zur Präzisierung der Bescheinigung; Adam Gegenbauer verlangte „wegen zum 9 Ämbter abcopierten Musicalien"142 laut der ersten Bescheinigung $23 \mathrm{fl} 57$ x, bei der zweiten $22 \mathrm{fl} 29$ x und der dritten $25 \mathrm{fl} 38$ x. Das heißt, dass für die neubeschaffte Musik, die für 27 Hochämter bestimmt war, insgesamt $72 \mathrm{fl} 4 \mathrm{x}$ ausbezahlt wurden.

Gleichen Betreff benutzte ebenfalls sein Nachfolger Franz Xaver Riesch, der für diese Dienste von 1755 bis 1779 zuständig war. Außer den üblichen Ausgaben für seine Tätigkeit tauchten auch zwei besondere Anlässe auf - im Jahre 1764 wurde er für drei Pumpermetten (samt der Buchbindergebühr) mit 37 fl 36 x entlohnt. ${ }^{143}$ Zweitens erstellte Riesch im Jahre 1775 laut der Verordnung vom 1. August „4 Catalogos über die vorhandenen Musicalien" für 25 fl 36 x. ${ }^{144}$ Dies könnte zirka 440 Folios entsprechen. Dies

136 Johann Adam Gegenbauer hatte die Kopistenstelle von 1742 bis 1753 inne, überdies war er als Subcantor und Violinist tätig.

137 Auch der erste Kornettist Joseph Griesbacher war als Kopist tätig, jedoch nicht für Zwecke der Domkapelle. Diese Tatsache geht aus einer Quittung hervor, die in den Beständen des Stifts St. Dorothea überliefert ist. Siehe Kapitel Preise der Kopien.

138 AT-DAWST, KMA, Rechnungsbuch 1716, Fol. 52r.

139 AT-DAWST, KMA, Rechnungsbuch 1717, Fol. 57v; Ibidem 1718 Fol. 54v.

140 AT-DAWST, KMA, Rechnungsbuch 1735, Fol. 56r.

$141 \mathrm{Zu}$ den weiteren Möglichkeiten der Musikalienübergabe und -erwerb der Kirchen siehe: FUKAČ, Jiř́. Křižovnický hudebni inventár̆: př́spěvek k poznáni križoonické hudebni kultury a jejího mista v hudebním životě barokni Prahy. Diplomarbeit, Masaryk-Universität, 1959.

142 AT-DAWST, KMA, Rechnungsbuch 1753, Fol. 88r.

143 AT-DAWST, KMA, Rechnungsbuch 1764, Fol. 134v.

144 AT-DAWST, KMA, Rechnungsbuch 1775, Fol. 78r. 
illustriert die Bemühungen einzelner Institutionen um Erfassung der zu der Kirche gehörigen Musikalien und damit verbundene Sicherung des eigenen Notenmaterials. ${ }^{145}$ Vom Magistrat kam die Verordnung, die eine Möglichkeit forderten, den Zustand und die eingekaufte Menge der Musikalien zu prüfen. ${ }^{146}$ Durch eine Erfassung der Noten anhand bestimmter Merkmale wurde die Kontrolle des eingekauften Materials vereinfacht und schneller durchführbar.

Die Kopisten kopierten nicht nur Figuralmusik - Joseph Anton Neck erhielt am 17. Juli 1728 wegen "Schreibung eines Choral Buches“ 24 fl. ${ }^{147}$ Im Jahre 1761 mussten die Choralbücher ausgebessert und neu geschrieben werden, was Joseph Neck für 13 fl durchführte. ${ }^{148}$ Die Choralbücher befanden sich wie die Musikinstrumente auf dem Musikchor. ${ }^{149}$

\section{Musikalien für die Gnadenbildkapelle}

Dem Gnadenbildensemble standen zur Verfügung eigene Musikalien, die aus den Stiftungsgeldern angeschafft worden sind. Die Musikalien für die Gottesdienste bei dem Gnadenbild wurden nicht alljährlich besorgt, sondern nur in ein paar Zeitspannen. ${ }^{150}$ Sie wurden in gleichem Jahr auch von mehreren Kopisten verfertigt; vom 1702 bis 1714 wurde Kopist Lambert Graf für die Kopien besoldet, im Jahre 1712 kam dazu Georg Arnold Schmidt und Geld erhielt ebenso der Kapellmeister Georg Reutter. Weiter wurde als Kopist der Fagottist Joseph Graf zuständig, in seiner Zeit erfolgten Zahlungen an die Kapellmeister. Zwischen 1750 und 1753 verrichtete die Dienste Adam Gegenbauer; als letzter Kopist kommt dann oben erwähnter Franz Xaver Riesch 1755 vor.

Einen erheblicheren Betrag von 170 fl nahm Ferdinand Schmid im Jahre 1743 an, der im gleichen Jahr zum Kapellmeister der Gnadenbildkapelle ernannt wurde. Ein Jahr danach bekam die Witwe nach Reinhardt $78 \mathrm{fl}$ 24x für bei ihr vorhandene, zum Kopieren

145 Diese Verordnung für eine eigenständige Erschließung der Musikalien und die Existenz der Musikalienkataloge könnte teilweise eine Erklärung bieten, warum sich in den Inventaren des Domeigentums noch im keine offiziellen Musikalienverzeichnisse bis ins Jahr 1799 befinden. Sie widerspiegelt aber den Zeitgeist - bei Verfertigung solcher Evidenzen musste somit die Domkirche zu Institutionen gehören, die die Musikalien versuchten zu erfassen. Es ist nicht eindeutig, ob sich um Inventare inkl. Incipitsangaben handelte. Ähnliche Tendenz lässt sich auch an anderen Orten nachvollziehen; um die Mitte des 18. Jahrhunderts sind Inventare aus Vorau, später aus Melk (AT), 1787 überliefert, zu den thematischen Inventaren gehören beispielsweise: das Musikalien-Inventar der Kirche zu St. Jakob Brünn (CZ), 1763; Musikinventar der Benediktiner in Raigern (CZ), 1771.

146 Sehr ähnliche Verordnung ist von der Esterhaz'schen Kapelle bekannt. Außer des Auftrags die Musikalien zu verzeichnen, gab es sehr genaue Anweisungen, die die Erfassung erfolgen sollte. Vgl. BARTHA, Dénes (Hrsg.). Haydn, Joseph. Gesammelte Briefe und Aufzeichnungen. Kassel: Bärenreiter, 1965, S. 49-51.

147 AT-DAWST, KMA, Rechnungsbuch 1728, Fol. 50r.

148 AT-DAWST, KMA, Rechnungsbuch 1761, Fol. 103v.

149 Vgl. die oben genannte Inventare des Kircheneigentums.

150 Es ging um die Zeitspannen 1709-1714, 1728-1730 und 1742-1755. Vgl. HOCHRADNER - VÖSÖSMARTY, op. cit., S. 156-164. 
erlaubte Musikalien. ${ }^{151}$ Im 1746 wurde wieder der Kapellmeister Schmid für Musikalien bezahlt - diesmal jedoch „wegen neu geschriebenen musicalien.“"152 Im Zeitraum 1. Dezember 1750 bis dahin 1751 wurde insgesamt für neue Musikalien 278 fl 20x bezahlt; zuerst wurde dem Kapellmeister Schmid mit 28 fl für neu geschriebene Musikalien bezahlt, danach folgt eine die Zahlung von $200 \mathrm{fl}$ an ihn „wegen neu componierten Musicalien zum Gnadenbild"153; weiteren 50 fl 20x bekam der Kopist Gegenbauer.

Solche Ausgaben könnten einer der Gründe sein, weshalb es der Stadtrat am 8. Juni 1757 veranlasste, einen Katalog zu den Gnadenbildkapelle zugehörigen Musikalien zu errichten. Für den errichteten Katalog wurde dem Bassisten-Accessisten Franz Schallhaß mit 8 fl 20x belohnt. Leider wurde nicht erwähnt, ob es sich um einen thematischen Katalog handelte. Die gesamte Musikalienmenge könnte ein Grund dafür sein, dass es nach 1755 keine Ausgaben auf Musikalien für die Verwendung in der Gnadenbildkapelle finden lassen.

\section{Preise der Kopien}

Wenn die Ausgaben auf die neu besorgten und verbesserten Musikalien während des 18. Jahrhunderts betrachtet werden, ergibt sich, dass die Ausgaben nicht gleichmäßig waren. Für die Essentialkapelle überschritten bis in das Jahr 1734 sie nur selten $100 \mathrm{fl}$ jährlich. ${ }^{154}$

In den 40er und 50er Jahren bewegten sich die Ausgaben durchschnittlich gegen $107 \mathrm{fl}$ pro Jahr. Am Ende der 60er sanken die Kosten nur zu $50 \mathrm{fl}$, dagegen im Jahre 1771 kam es zu einem markanten Anstieg - denn es wurde das Vierfache für die Kopiaturen ausbezahlt. Durch die vorliegende Grafik wird die deutliche Veränderung nach dem Tod des Kapellmeisters Georg von Reutter und Beginn der Tätigkeit Leopold Hofmanns ersichtlich. Nach dem Rückgang der Ausgaben kommt erst nach dem Eintritt von Johann Georg Albrechtsberger im Jahre 1793 zu erneut erheblichen Ausgaben für neu geschriebene Musikalien. ${ }^{155}$

Wünschenswert wäre es, wenn die Angaben in den Rechnungsbüchern präziser vermerkt würden, beispielsweise eine genaue Anzahl der Kompositionen oder Gattungen angeben würde. Einen Einblick in die Preise von geschriebenen Musikalien bringen jedoch sporadisch erhaltene Quellen zum Musikbetrieb in anderen Kirchen. Vom 11. Oktober 1756 stammt eine Quittung des Kopisten Joseph Griesbacher, der ab dem Jahre 1768 als Kornettist bei St. Stephan belegt ist. Für zwei Litaneien und drei Stück Salve Regina, die insgesamt auf 61 Bögen geschrieben wurden, verrechnete er dem Herrn Prälat

151 AT-DAWST, KMA, Rechnungsbuch 1743, Fol. 108v.

152 AT-DAWST, KMA, Rechnungsbuch 1746, Fol. 83r.

153 WStLA, HS, A/41.25 Inventar von St. Stephan 1751, Fol 114v.

154 Die obenerwähnte Zahlung von 732 fl. an den Kapellmeister Reutter wurde nicht in diese Durchschnittsberechnung eingeschlossen.

155 Zwischen den Jahren 1788 und 1791 sind keine Kirchenrechnungen vorhanden. 


\section{Ausgaben für Musikalien}

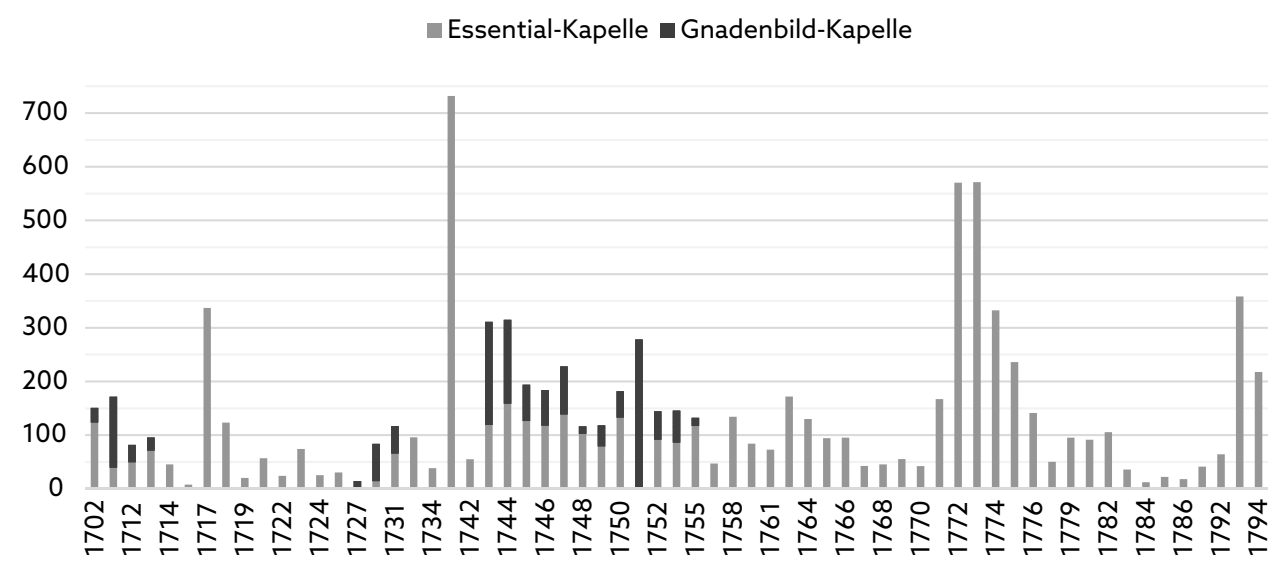

Grafik 2 Ausgaben für Musikalien im Stephansdom

von St. Dorothea durch den Regens Chori den Betrag 5 fl 5x (d. h. 5x pro Bogen). ${ }^{156}$ Als ein anderes Beispiel kann die Bemerkung im Brünner St.Jakob-Musikinventar dienen; im Jahre 1763 wurde vermerkt, dass für die Stadtpfarrkirche in Brünn drei besorgte Vesperkompositionen von Ägidius Schenk, Johann Georg Albrechtsberger und Christian Widmann insgesamt für $4 \mathrm{fl} 10 \mathrm{x}$ erkauft wurden. ${ }^{157}$ Die Bogenanzahl der in der Sammlung bis heute erhaltenen Musikalien nach entspricht der Preis den vorher genannten 5x pro Bogen (verrechnet inkl. der Umschläge). Die Taxe im Dom war bereits 1743 höher als in den genannten Beispielen. Die Zahlung an Reinhards Witwe belegt, dass die vereinbarte Taxe für einen Bogen $7 \mathrm{x}$ war. Bei der Essentialkapelle kommt die erste Taxenangabe von $7 \mathrm{x}$ pro Bogen im Jahre 1762 ausdrücklich vor. ${ }^{158}$

Wie sich die Preise weiter entwickelten, veranschaulicht ein Gesuch des Kopisten Jakob Nurscher; am 30. April 1809 bat er das Kirchenmeisteramt um Erhöhung des Pauschalbetrags für das Musikalienkopieren. Er führte an, dass er bereits 20 Jahre die Musikalien ohne Erhöhung des Papiergeldes kopiert. Für das Kopieren eines Bogens wurde ihm die ganze Zeit hindurch $7 \mathrm{x}$ bezahlt, indem die Unkosten für das nötige Papier und Tinte eingeschlossen waren. Als genügendes Argument für die Zustimmung des Amtes war, dass der Papier- und Materialienpreis merklich gestiegen ist. Die Erhöhung des Pauschalbetrags wurde vom Kirchenmeister Franz Siegl unterstützt; er fügte

156 Vgl. Stiftsarchiv Klosterneuburg, Bestand Stift St. Dorothea, Sign. D145, Nr. 2.

157 Bei keinem anderen Eintrag wurde der Preis vermerkt. Für weitere Informationen zum St.-Jakob-Inventar siehe: RATOLÍSTKOVÁ, Michaela - SPÁČILOVÁ, Jana. The music collection of Brno St. James regenschori Matthaeus Rusmann and its inventory from 1763. In Musicologica Brunennsia, 53, 2018, Nr. 2, S. 201-219. KRAMÁŘOVÁ, Helena. Neu besorgte Musikalien für die Stadtpfarrkirche St. Jakob in Brünn zwischen den Jahren 1763 und 1781. In Musicologica Brunensia, Masarykova univerzita, 2018, roč. 53, č. 2, s. 235-265.

158 AT-DAWST, KMA, Rechnungsbuch 1762, Fol. 103v. 
hinzu, dass Jakob Nurscher immer verlässlich war und seine Dienste gut leistete. Ab 1809 sollte er deshalb pro Bogen $12 \mathrm{x}$ bekommen, wobei drinnen wieder das Material inkludiert wurde. ${ }^{159}$

\section{Resümee}

Die Quellen zur Musikpflege am Stephansdom ermöglichen eine Einsicht in das Musikgeschehen im Wiener Dom während des 18. Jahrhunderts, wie sie in dieser Form und Menge für die Wiener Kirchenmusik-Situation dieser Zeit fast unvergleichlich ist. Hohe Ansprüche an die Musiker, sowie ein reges liturgisches Geschehen verlangten die Konstituierung zweier Musikensembles, damit alle notwendigen Musikdienste wahrgenommen werden konnten. Der Anstieg an beschäftigten Musikern war dank errichteter Stiftungen möglich. Die meisten Ausgaben für die Musik übernahm aber trotz dieser nach wie vor die vom Magistrat verwaltete Kirchenkassa, weshalb das eigentliche Entscheidungsorgan über die Dommusikkapelle der Stadtrat war. Wegen der notwendigen Eingaben an ihn, seiner Erledigungen und seiner Kontrolle sind im einschlägigen Aktenmaterial zahlreiche Dokumente erhalten, die die Anschaffung von Musikinstrumenten und auch (freilich nicht ganz detailliert) von Musikalien belegen. Mithilfe des zitierten Scheibens von Johann Georg Albrechtsberger kann belegt werden, dass auch die Westempore im Dom zur Aufführung von Figuralmusik - obschon nur bei speziellen Anlässen und unter Sonderbedingungen - diente.

\section{Bibliography}

\section{Sources}

Wiener Stadt- und Landesarchiv (weiter nur: AT-WStLA), Handschriften A41/20. Kirchenmeisteramts-Rechnungen von St. Stephan -1702 .

AT-WStLA, Handschriften A41/25 Kirchenmeisteramts-Rechnungen von St. Stephan - 1750-1751.

AT-WStLA, Handschriften A41/28 Kirchenmeisteramts-Rechnungen von St. Stephan - 1781.

AT-WStLA, Handschriften A41/29 Kirchenmeisteramts-Rechnungen von St. Stephan - 1792.

AT-WStLA, Hauptarchiv, Akten und Verträge: A1/9/1707, Testament des hofbefreiten Handelsmannes Michael Kurz.

AT-WStLA, Stadtarchiv, Historische Registratur, A6/2 Messstiftungen: 2.14/1 Andre Hüttauer (1718-1772), 2.14/2 Andre Hüttauer.

AT-WStLA, Handschriften, A42/2 Inventar von St. Stephan 1732.

AT-WStLA, Handschriften, A42/3 Inventar von St. Stephan 1742.

Stiftsarchiv Klosterneuburg, Bestand Stift St. Dorothea, Sign. D145.

159 AT-DAWST, KMA, Erledigungen 1808-1811, Kart. 11/1, Fol. 1451. 
Domarchiv St. Stephan (weiter: AT-DAWST), Kirchenmeisteramt von St. Stephan (KMA), Inventarium 1768.

AT-DAWST, KMA, Inventarium 1771.

AT-DAWST, KMA, Erledigungen 1808-1811, Kart. 11/1.

AT-DAWST, KMA, Erledigungen 1800-1804, Kart. 9/1.

AT-DAWST, KMA, Erledigungen 1792-1800, Kart. 8/1.

AT-DAWST, KMA, Erledigungen 1792-1800, Kart. 8/2.

AT-DAWST, KMA, Erledigungen 1792-1800, Kart. 8/3,

AT-DAWST, KMA, Erledigungen 1786-1792, Kart. 7a/1.

AT-DAWST, KMA, Erledigungen 1786-1792, Kart. 7a/2.

AT-DAWST, KMA, Erledigungen 1771-1786, Kart. 7/2.

AT-DAWST, KMA, Erledigungen 1804-1808, Kart. 10/2.

AT-DAWST, KMA, Rechnungsbuch 1714.

AT-DAWST, KMA, Rechnungsbuch 1716.

AT-DAWST, KMA, Rechnungsbuch 1717.

AT-DAWST, KMA, Rechnungsbuch 1718.

AT-DAWST, KMA, Rechnungsbuch 1721.

AT-DAWST, KMA, Rechnungsbuch 1722.

AT-DAWST, KMA, Rechnungsbuch 1727.

AT-DAWST, KMA, Rechnungsbuch 1728.

AT-DAWST, KMA, Rechnungsbuch 1730.

AT-DAWST, KMA, Rechnungsbuch 1735.

AT-DAWST, KMA, Rechnungsbuch 1736.

AT-DAWST, KMA, Rechnungsbuch 1743.

AT-DAWST, KMA, Rechnungsbuch 1745.

AT-DAWST, KMA, Rechnungsbuch 1746.

AT-DAWST, KMA, Rechnungsbuch 1747.

AT-DAWST, KMA, Rechnungsbuch 1748.

AT-DAWST, KMA, Rechnungsbuch 1750.

AT-DAWST, KMA, Rechnungsbuch 1753.

AT-DAWST, KMA, Rechnungsbuch 1755.

AT-DAWST, KMA, Rechnungsbuch 1757.

AT-DAWST, KMA, Rechnungsbuch 1758.

AT-DAWST, KMA, Rechnungsbuch 1761.

AT-DAWST, KMA, Rechnungsbuch 1762.

AT-DAWST, KMA, Rechnungsbuch 1764.

AT-DAWST, KMA, Rechnungsbuch 1765.

AT-DAWST, KMA, Rechnungsbuch 1766.

AT-DAWST, KMA, Rechnungsbuch 1767.

AT-DAWST, KMA, Rechnungsbuch 1768.

AT-DAWST, KMA, Rechnungsbuch 1770.

AT-DAWST, KMA, Rechnungsbuch 1772.

AT-DAWST, KMA, Rechnungsbuch 1774.

AT-DAWST, KMA, Rechnungsbuch 1775.

AT-DAWST, KMA, Rechnungsbuch 1779.

AT-DAWST, KMA, Rechnungsbuch 1782.

AT-DAWST, KMA, Rechnungsbuch 1783. 
AT-DAWST, KMA, Rechnungsbuch 1785.

AT-DAWST, KMA, Rechnungsbuch 1793.

AT-DAWST, KMA, Rechnungsbuch 1794.

\section{Literature}

ALLMER, Gottfried. Orgeln und Orgelbau in der Dom- und Metropolitankirche St. Stephan in Wien. In Das Orgelforum 14, 2011, S. 32-67.

BARTHA, Dénes (Hrsg.). Haydn, Joseph. Gesammelte Briefe und Aufzeichnungen. Kassel: Bärenreiter, 1965.

BRIX, Michael. Trauergerüste für die Habsburger in Wien. In Wiener Jahrbuch Für Kunstgeschichte, 26(1), 1973.

BRUNNER, Hans. Die Kantorei bei St. Stephan in Wien. Wien. Verl. Albrecht Dürer, 1948.

CZEIKE, Felix. Historisches Lexikon Wien. Wien: Verlag Kremayr \& Scheriau, 1994.

D'ELVERT, Christian. Geschichte der Musik in Mähren und Oesterr.-Schlesien. Brno, 1873.

DICKEY, Bruce. Far buon stromento: A Survey of the Technique and Repertoire of the Cornetto in its Golden Age. In HAAG, Sabine. (Hrsg.). Die Zinken und der Serpent der Sammlung Alter Musikinstrumente. Bergkirchen, Edition Bochinsky, 2011, S. 68-89.

EBENBAUER, Melitta. Zur Geschichte der Dommusik. [online] URL: < http://www.dommusik-wien. at/Dommusik/media/ZurGeschichtederDommusik_Ebenbauer.pdf $>$

FUKAČ, Jiří. Křižovnický hudebni inventár̆: přispěvek $k$ poznáni kř́žounické hudebni kultury a jejiho mista v hudebním životě barokni Prahy. Diplomarbeit, Masaryk-Universität, 1959.

Funftzig-Jähriges Jubel-Fest des wunderthätigen Gnaden-Bilds Mariae von Pötsch [...] Wien, 1747 (ÖNB 74.632-B).

HASELBÖCK, Hans. Sechs Jahrhunderte Orgelbau im Wiener Stephansdom. In HASELBÖCK, Hans. Von der Orgel und der Musica Sacra. Historisch-kritische Beiträge zu Fragen von Orgelbau, Orgelkomposition und neuer Kirchenmusik. Wien-München, 1988.

HERSCHE, Peter. Muße und Verschwendung: Europäische Gesellschaft und Kultur im Barockzeitalter. Freiburg, Herder Verlag, 2006.

HILSCHER, Elisabeth. Musikbruderschaften als Karrierenetzwerke für Stadt und Hof in Wien. In LOBENWEIN, Elisabeth - SCHEUTZ, Martin - WEISS, Alfred Stefan (Hg.). Bruderschaften als multifunktionale Dienstleister der Frühen Neuzeit in Zentraleuropa. Wien: Böhlau, 2018, S. 357-372.

HOCHRADNER, Thomas - VÖSÖMARTY, Géza Michael. Zur Musikpflege am Altar Mária Pócs (Maria Pötsch) in St. Stephan in Wien. In Studia Musicologica Academiae Scientiarum Hungaricae, 2000, T. 41, Fasc. 1/3, S. 133-175.

HOPFNER, Rudolf. Hencke (Henke, Henckl, Hengel), Johann. In Oesterreichisches Musiklexikon online [online], 20XX [2019-03-13] URL: 〈http://www.musiklexikon.ac.at/ml/musik_H/Hencke_Johann.xml>.

HOPFNER, Rudolf. Thier (Thir, Thür, Dirr, Dürr), Familie. In Oesterreichisches Musiklexikon online [online], 20XX [2019-03-20] URL: 〈http://www.musiklexikon.ac.at/ml/musik_T/Thier_Familie.xml>.

KRAMÁŘOVÁ, Helena. Neu besorgte Musikalien für die Stadtpfarrkirche St. Jakob in Brünn zwischen den Jahren 1763 und 1781. Musicologica Brunensia, Masarykova univerzita, 2018, roč. 53, č. 2, s. 235-265.

SEIFERT, Herbert. Die Entfaltung des Barocks, In Flotzinger, Rudolf. (Hrsg.) Musikgeschichte Österreichs. Wien: Böhlau Verlag, 1995, 2. Aufl., Bd. 1, S. 299-361. 
SOMMER-MATHIS, Andrea - FRANKE, Daniela - RISATTI, Rudi. Spectacolo Barocco! Triumph des Theaters, Wien: Michael Imhof Verlag, 2017.

RATOLÍSTKOVÁ, Michaela - SPÁČILOVÁ, Jana. The music collection of Brno St. James regenschori Matthaeus Rusmann and its inventory from 1763. In Musicologica Brunennsia, 53, 2018, Nr. 2, S. 201-219.

ŠTĚDROŇ, Bohumír. Společenské úkoly hudby v 18. století. [Gesellschaftliche Funktionen der Musik im 18. Jahrhundert.] In Časopis Matice moravské 69, 1950.

ŠTĚDROŇ, Bohumír. Zur Entwicklungsgeschichte einer unbekannten Musik-Gesellschaft im 17. und 18. Jhdt. Die Musikforschung 21, 1968, s. 438-458.

UHLIRZ, Carl. Die Rechnungen des Kirchenmeisteramts von St. Stephan zu Wien. Wien: Braumüller, 1901-1902.

ZSCHOKKE, Hermann. Geschichte des Metropolitan-Capitels zum Heiligen Stephan in Wien (nach Archivalien). Wien: Verlag von Carl Konegen, 1895. 
